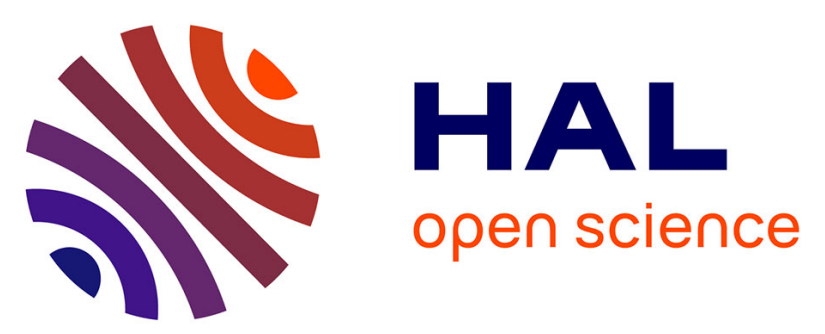

\title{
Exploring the excited-state dynamics of hydrocarbon radicals, biradicals, and carbenes using time-resolved photoelectron spectroscopy and field-induced surface hopping simulations
}

Anja Röder, Jens Petersen, Kevin Issler, Ingo Fischer, Roland Mitrić, Lionel Poisson

\section{To cite this version:}

Anja Röder, Jens Petersen, Kevin Issler, Ingo Fischer, Roland Mitrić, et al.. Exploring the excitedstate dynamics of hydrocarbon radicals, biradicals, and carbenes using time-resolved photoelectron spectroscopy and field-induced surface hopping simulations. Journal of Physical Chemistry A, 2019, 123 (50), pp.10643-10662. 10.1021/acs.jpca.9b06346 . hal-02440330

\author{
HAL Id: hal-02440330 \\ https://hal.science/hal-02440330
}

Submitted on 15 Jan 2020

HAL is a multi-disciplinary open access archive for the deposit and dissemination of scientific research documents, whether they are published or not. The documents may come from teaching and research institutions in France or abroad, or from public or private research centers.
L'archive ouverte pluridisciplinaire HAL, est destinée au dépôt et à la diffusion de documents scientifiques de niveau recherche, publiés ou non, émanant des établissements d'enseignement et de recherche français ou étrangers, des laboratoires publics ou privés. 


\section{Exploring the Excited-State Dynamics of Hydro-}

carbon Radicals, Biradicals and Carbenes using

\section{Time-Resolved Photoelectron Spectroscopy and}

\section{Field-Induced Surface Hopping Simulations}

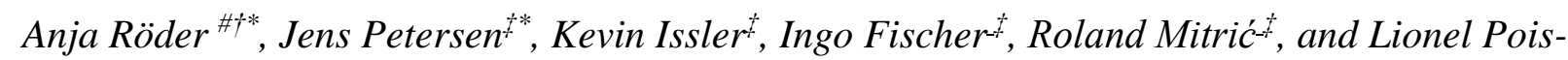
$\operatorname{son}^{\S}$

\# Present address differs from where the work was done

$\dagger$ Department of Chemistry, University of Ottawa, 150 Louis Pasteur, Ottawa, Ontario, Canada K1N 6N5

Institute of Physical and Theoretical Chemistry, University of Würzburg, Am Hubland, 97070 Würzburg, Germany

$\S$ LIDYL, CEA, CNRS, Université Paris-Saclay, CEA Saclay 91191 Gif-sur-Yvette France

AUTHOR INFORMATION

Corresponding Author

Anja Röder, aroder@uottawa.ca; Jens Petersen, jens.petersen@uni-wuerzburg.de 


\begin{abstract}
:
Reactive hydrocarbon molecules like radicals, biradicals and carbenes are not only key players in combustion processes and interstellar and atmospheric chemistry, but some of them are also important intermediates in organic synthesis. These systems typically possess many low-lying, strongly coupled electronic states. After light absorption, this leads to rich photodynamics characterized by a complex interplay of nuclear and electronic motion, which is still not comprehensively understood and not easy to investigate both experimentally and theoretically. In order to elucidate trends and contribute to a more general understanding, we here review our recent work on excitedstate dynamics of open-shell hydrocarbon species using time-resolved photoelectron spectroscopy and field-induced surface hopping simulations, and report new results on the excited-state dynamics of the tropyl and the 1-methylallyl radical. The different dynamics are compared, and the difficulties and future directions of time-resolved photoelectron spectroscopy and excited state dynamics simulations of open-shell hydrocarbon molecules are discussed.
\end{abstract}




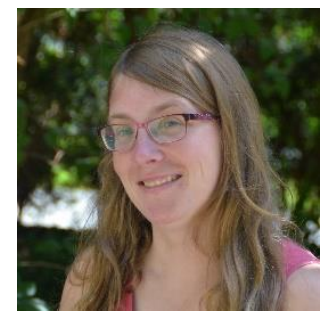

Anja Röder was born in 1988. After obtaining her joint Master of Science in Chemistry (Julius-Maximilians-Universität Würzburg) and chemical engineering diploma (École Supérieure de Chimie Physique Électronique de Lyon), she did her doctoral work under the guidance of Ingo Fischer (University of Würzburg) and Lionel Poisson (Université de Paris-Sud) on the time-resolved photoelectron spectroscopy of open-shell species, where she obtained her $\mathrm{PhD}$ in 2017. She was awarded several prices for her thesis, including the doctoral prize of the institute of Chemistry of the University of Würzburg and the award of excellence in theoretical, physical or analytical chemistry of the French Chemical Society. Since 2018 she is working as a postdoctoral associate with Albert Stolow in Ottawa and continues her research there as a Humboldt fellow since 2019. Her research areas are in the field of chemical dynamics, focusing on time-resolved photoelectron spectroscopy, absolute photoionization cross sections and timeresolved X-ray absorption and emission spectra.

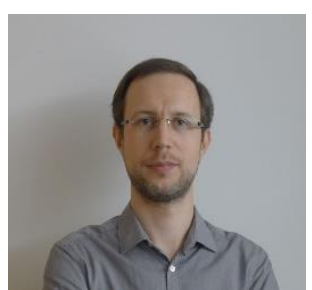

Jens Petersen was born in 1983 in Berlin. After studying chemistry at HumboldtUniversität zu Berlin he did his doctoral work with Vlasta Bonacic-Koutecky and Roland Mitric and received his $\mathrm{PhD}$ in theoretical physics from Freie Universität Berlin in 2013. For his thesis, he was awarded the Tiburtius prize of the Berlin Universities. He has been working since as a senior scientist in the group of Roland Mitric at the Julius-Maximilians-Universität Würzburg. His research interests include the development of methods for the simulation of coupled electron-nuclear dynamics in molecular systems and the application to nonadiabatic photodynamics, time-resolved spectroscopy and optimal quantum control.

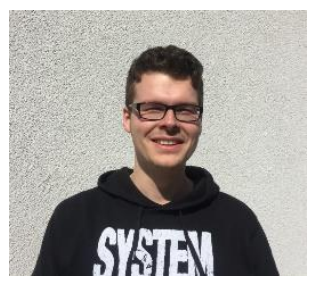

Kevin Issler was born in 1994. He obtained his Master of Science in Chemistry from the Julius-Maximilians-Universität Würzburg and started his $\mathrm{PhD}$ in 2019 in the group of Roland Mitric. His research focuses on the quantum-classical simulation of ultrafast nonadiabatic photodynamics and time-resolved spectroscopy as well as the development of methods for the simulation of ionization processes.

Ingo Fischer obtained a diploma degree 1989 in chemistry from the University of Bonn and Ph.D from the TU München in 1992, working under the guidance of Vladimir Bondybey. After postdoctoral work with Albert Stolow at the National Research Council in Ottawa from 1993-1995 he joined the chemistry department of the ETH Zürich, being associated with the group of Peter Chen. Since 2001 he is a Professor of Physical Chemistry at the University of Wuerzburg. His main research interest are the chemistry and chemical dynamics of reactive intermediates, photoionization and photoelectron spectroscopy as well as the timeresolved spectroscopy of polycyclic aromatic hydrocarbons and their clusters.

Roland Mitric obtained his $\mathrm{PhD}$ in physical and theoretical chemistry from the Humboldt-Universität zu Berlin in 2003 working with Vlasta Bonacic-Koutecky. For his thesis he was awarded the Fischer-Nernst Prize and the Adlershofer Prize. From 2009-2013 he was an Emmy-Noether fellow at the Physics Department of the Freie Universität Berlin leading a junior research group. Since 2013 he is a full professor and chair for theoretical chemistry at the Julius-Maximilians-Universität Würzburg. In 2015 he received a European Research Council (ERC) consolidator grant. His research directions include the development of methods for the simulation of light-induced nonadiabatic dynamics in complex molecular systems, development of efficient quantum chemical methods as well as applications to the study of energy and charge 
transport properties in supramolecular systems, photochemistry and photophysics of complex molecules, (photo)catalysis and cluster physics and chemistry. He is author of more than 150 publications.

Lionel Poisson is a former student from the Ecole Normale Supérieure, Paris. He received his Ph.D from Université Paris-Sud in 2001, under the supervision of Dr. Jean-Michel Mestdagh. He worked two years as a postdoc in the groups of Pr. Daniel Neumark and Pr. Steven Leone before being hired by CNRS in Saclay. $\mathrm{He}$ is currently senior researcher and leads the Reaction Dynamics group of LIDYL, a laboratory of the Université Paris-Saclay. His research interest are the relaxation dynamics of isolated and solvated species, dynamics of and on rare gas clusters as well as the ionization dynamics.

\section{Introduction}

Investigating excited-state dynamics of isolated molecules over a wide range of time-scales has sparked an enormous interest, as indicated by the award of a Nobel prize in chemistry to A. Zewail in $1999^{1}$. The vast majority of studies investigated closed-shell systems, as they are often easily available. Studies on the excited-state dynamics of hydrocarbons with open-shell or carbene character, however, are scarce, although these may provide valuable insights in these fascinating species: (a) From an organic chemist's viewpoint, such molecules are very reactive and readily form new bonds, hence their involvement as intermediates in combustion ${ }^{2,3}$ as well as interstellar ${ }^{4,5}$ and atmospheric chemistry ${ }^{6}$. (b) From a physical chemist's viewpoint these species possess low-lying electronic states, which are often strongly coupled, leading to quite complex electronic spectra and intricate dynamical processes induced upon photoexcitation. (c) From a theoretical chemist's viewpoint, calculations on hydrocarbons with open shell or carbene character incorporate several challenges such as non-adiabatic effects, spin contamination, or the presence of low-lying, diffuse Rydberg states ${ }^{7}$. This requires a careful choice of the appropriate quantum chemical method, and highly correlated multi-reference methods may be mandatory in certain cases. Investigating the excited-state dynamics of hydrocarbons with open shell or carbene character is therefore a challenging task both theoretically and experimentally. Due to their high reactivity, these species must 
be produced in situ in the gas phase from suitable precursors, as they are mostly too reactive to be isolated under normal conditions. Finding suitable precursors for a clean and efficient production is demanding and difficult. On the other hand (b) implies that the first excited state often lies in the visible and higher-lying excited states not far above, often at wavelengths easily accessible experimentally.

In our quest to understand the excited-state dynamics of hydrocarbons with open shell or carbene character, we investigate the following questions: (1) how do their excited-states couple and how fast do they deactivate? (2) Can we link electronic structure to dynamics/coupling for these unusual electronic configurations? To answer these questions, we require an experimental method that is sensitive to the character of the electronic states involved in the dynamics and at the same time suitable to detect molecules at low concentrations. Time-resolved-photoelectron spectroscopy (TRPES) of valence electrons has been the method of choice to investigate the excited-state dynamics of many closed-shell molecules and anions ${ }^{8-12}$, as it offers several advantages:

- Different electronic states can be disentangled through their characteristic photoelectron spectra.

- Time-independent signals can be easily identified and attributed in TRPES as well as subtracted from the final spectra; it therefore provides a background-free measurement.

- Different orders of ionization processes (e.g. [1+1'] vs [1+2'] or [1+3']) can be readily identified in the photoelectron spectra, which is not possible in other methods relying on ionization as the detection step, as e.g. in time-resolved mass spectrometry. 
- $\quad$ Provided that the probe photon energy is high enough, all states can be ionized, and even poorly fluorescent states can be probed.

TRPES is even more favorable for open-shell species, since these tend to have lower ionization energies (IE) than their closed-shell counterparts. The benzyl radical, for example, has an IE of $7.24 \mathrm{eV}^{13,14}$, while the IE of toluene is more than $1 \mathrm{eV}$ higher $\left(8.828 \mathrm{eV}^{15}\right)$. For the tert-butyl radical, the IE is even $4 \mathrm{eV}$ lower than for its closed-shell analog isobutane (6.58 vs. $\left.10.57 \mathrm{eV}^{16}\right)$. Similar trends can be observed for the position of the excited states: While in small closed-shell species the first excited state is often found in the UV range, for many radicals the first excitation energy is markedly lower, e.g. $2.73 \mathrm{eV}$ for benzyl ${ }^{17}$ vs. $4.65 \mathrm{eV}$ for toluene ${ }^{18}$, or $3.7 \mathrm{eV}$ for tertbutyl $^{19}$ vs. $8.6 \mathrm{eV}$ for isobutane ${ }^{20}$. Therefore, UV light may likely populate the $2^{\text {nd }}$ or higher excited states $^{21}$. In contrast to closed-shell molecules, in open-shell systems such states are frequently already of Rydberg character. While in resonance stabilized radicals ${ }^{21}$, this leads to the simultaneous presence of Rydberg and low-lying valence excited states (often of $\pi \pi^{*}$ or $n \pi *$ character), for saturated species such as alkyl radicals, with the unpaired electron situated in a carbon $2 p$ orbital, the lowest lying excited states exclusively possess Rydberg character (see examples below). Investigating the UV photodynamics of open-shell molecules therefore not only allows one to obtain information on relaxation time scales but also to elucidate the intricate interplay between several coupled excited states.

In the following we will review our work on the TRPES and nonadiabatic dynamics simulations of hydrocarbons with open-shell or carbene character, including previously unpublished results, with the aim to show the still open questions and to emphasize the diversity of information one can glean from their TRPES. 


\section{Experimental and Theoretical Methods}

A prerequisite to record TRPES spectra of hydrocarbons with open shell or carbene character is a clean production. Even though mass-selected TRPES have been recorded for closed-shell molecules previously ${ }^{22-24}$, this technique had not yet been applied to the dynamics of reactive intermediates before we started in 2006. Different kinds of external stresses can lead to breaking of specific bonds in a precursor, however, selectivity and conversion rates vary according to each method and precursor. While photolysis ${ }^{25}$ and electrical discharges ${ }^{26}$ have been successfully applied to generate reactive species, in all our experiments described below pyrolysis ${ }^{27}$ has been used as the production method. In a nutshell, a diluted precursor-noble gas beam (seeded beam) passes through a resistively heated silicon carbide $(\mathrm{SiC})$ tube, which reaches temperatures up to $1000{ }^{\circ} \mathrm{C}$. The $\mathrm{SiC}$ tube acts as a microreactor, where the precursor molecules collide with the wall or with fast carrier gas molecules, thus breaking the weakest bond(s). Through careful temperature tuning of the $\mathrm{SiC}$ tube and a suitable precursor design, a full conversion of the precursor to the desired species can be obtained, allowing one to measure TRPES of the latter.

The reactive species are then expanded in a supersonic jet and cooled to a vibrational temperature $\mathrm{T}_{\mathrm{vib}}$ of approximately $150 \mathrm{~K}^{28}$, resulting in collision-free conditions for the laser experiments. The ions and electrons are created using the pump-probe technique, where a first laser excites the molecules (pump) and a second laser ionizes them (probe); the temporal resolution is obtained by changing the time-delay between both. Several different techniques exist to examine the created ions and electrons. As for time-resolved experiments on molecules with open shell or carbene character, it is paramount to control whether the desired species has been created; mass spectroscopy provides a first identification. 
Photoelectron spectra can be recorded using either magnetic bottles ${ }^{8,29}$, velocity-map imaging techniques $\mathrm{s}^{30-32}$ or combinations of the $\mathrm{two}^{33}$. In magnetic bottle experiments, an inhomogeneous magnetic field rapidly parallelizes electron trajectories to a flight tube, and a constant magnetic field guides the electrons in a helical motion toward to the detector. The kinetic energy of the photoelectrons is then inversely proportional to the square of the arrival time of the electrons. Such a technique, however, does not allow the measurement of the angular distribution of the emitted photoelectrons.

Most experiments discussed below rely on velocity-map imaging $(\mathrm{VMI})^{32}$. In these experiments an electrostatic lens is used to project the emitted electron clouds on a 2D detector, which typically consists of two multi-channel plates coupled to a phosphor screen. Each time charged particles hit the MCP, the phosphor screen emits light, which is then recorded by a CCD camera. The electron clouds are actually 3D spheres, where electrons of different kinetic energies correspond to spheres with different radii. To recover the full 3D spheres from these 2D-dimensional images, inversion algorithms can be applied - provided that the ionizing laser is polarized parallel to the detector plane. Though several inversion algorithms ${ }^{34-37}$ exist, in all experiments reviewed here the $\mathrm{pBasex}$ algorithm ${ }^{38}$ was used. Velocity map imaging allows easy access to the photoelectron angular distribution (PAD). For ionization with $\mathrm{k}$ linearly polarized photons, the angle-dependent intensity $I(\theta)$ reads

$$
I(\theta) \propto 1+\sum_{n=1}^{k} \beta_{2 n} P_{2 n}(\cos \theta)
$$

where $\beta_{2 n}$ is the anisotropy parameter of order $2 n$, and $P_{2 n}(\cos \theta)$ a Legendre polynomial. In the case of one-photon ionization ( $\mathrm{k}=1)$, the PAD will be characterized by only $\beta_{2}$, which can vary between 2 and $-1^{39}$. This value depends on a multitude of parameters, including the character 
of the electronic state, which is ionized as well as the photoionization energy. Therefore, a straightforward interpretation of the $\beta_{2 n}$ parameters is rarely possible, but they may provide further consolidation of a preexisting hypothesis. For more details about TRPES the reader is referred to ${ }^{8,32,40}$, and for a detailed discussion of PADs to ${ }^{41}$.

For the theoretical simulation of the photodynamics of open-shell species, we have utilized our previously developed field-induced surface-hopping method (FISH) ${ }^{42}$, which has been successfully employed to investigate dynamical processes in a variety of closed-shell molecules ${ }^{43-49}$. Briefly, the idea of the FISH method is to propagate the nuclei of the molecular system classically "on the fly" in a specified electronic state and to allow for state switches according to a quantum mechanically calculated hopping probability. The latter is based on solving the time-dependent Schrödinger equation for the electronic degrees of freedom along the classical trajectories,

$$
i \hbar \dot{c}_{i}(t)=E_{i}[\boldsymbol{R}(t)] c_{i}(t)-\sum_{j \neq i}\left(i \hbar \boldsymbol{d}_{i j}[\boldsymbol{R}(t)] \cdot \dot{\boldsymbol{R}}(t)+\boldsymbol{\mu}_{i j}[\boldsymbol{R}(t)] \cdot \boldsymbol{E}(t)\right) c_{j}(t)
$$

with the energy $E_{i}$ of the i-th electronic state, the nonadiabatic coupling $\boldsymbol{d}_{i j}$ and the electronic transition dipole moment $\boldsymbol{\mu}_{i j}$ depending on the nuclear trajectory $\boldsymbol{R}(t)$. The quantity $\boldsymbol{E}(t)$ describes the electric laser field interacting with the molecule, while the $c_{i}$ are electronic state coefficients whose absolute squares determine electronic state populations $\rho_{i i}=\left|c_{i}\right|^{2}$. These are subsequently used to calculate hopping probabilities according to

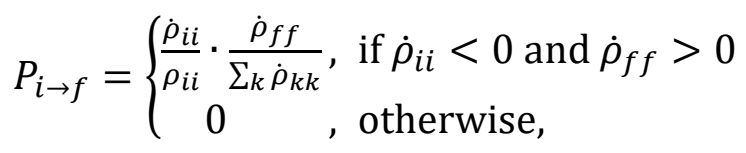

where the sum in the denominator is taken over all states $k$ whose population is growing $\left(\dot{\rho}_{k k}>\right.$ $0)$. The final decision for a state switch is then made in a stochastic process. 
The electronic energies and couplings necessary to solve Eq. (2), as well as the forces needed for the classical propagation of the nuclei are obtained from quantum chemical calculations. For the case of species with open shell or carbene character, a careful choice of the respective methods is mandatory to properly describe the electronic states of different character occurring in these systems, and at the same time allows for efficient computation. The variety of methods employed in the studies presented here reaches from semiempirical and density functional methods up to high-level ab initio approaches, in each case depending on the specific system under study.

The classical treatment of the nuclei requires the supply of initial coordinates and velocities prior to the dynamics simulation. These can be obtained either by sampling a quantum mechanical phase space distribution, e.g. the Wigner function ${ }^{50}$, for the harmonic normal modes of the system, or in a purely classical way from a long thermalized trajectory propagated in the electronic ground state. Both approaches have their pros and cons: Sampling of a Wigner distribution provides an adequate way to represent the initial quantum state of the system, but anharmonic effects, which may become important at higher temperatures, or for particular normal modes such as torsions, are neglected. These are in turn included when a classical trajectory is propagated "on the fly" on the proper electronic potential energy surface, but at the cost of the sampled distribution being narrower in phase space than the quantum mechanical one, not least due to the lack of zero-point energy. This problem is, however, less pronounced at higher temperatures, where the classical approximation becomes more adequate.

The number of initial conditions necessary to properly sample the quantum mechanical wavefunction depends on the specific aim of the simulation. While for the representation of low-probability processes (i.e. minor reaction channels, very low electronic state populations) or subtle temporal structures of the population dynamics, numbers well beyond 100 may be required, our 
experience with a variety of molecular systems has shown that in order to reproduce the major reaction pathways and the principal time scales of the electronic population dynamics, 50-100 initial conditions are usually sufficient. This is particularly true for low-dimensional systems, and we could show that e.g. for the silver cluster $\mathrm{Ag}_{3}$, actually less than 50 trajectories were enough to obtain converged results ${ }^{51}$.

To establish the connection to time-resolved photoionization experiments, different approaches can be followed. To obtain a simple estimate of the total photoionization efficiency, the IE from the currently populated electronic states can be calculated along each surface hopping trajectory, and ionization can be considered possible if the energy of the probe photon surpasses this energy. A more sophisticated approach consists in an approximate description of the ionization continuum by a dense manifold of discrete states, whose population due to the probe pulse is explicitly accounted for by numerical solution of the time-dependent Schrödinger equation ${ }^{51}$. In this way, the photoelectron kinetic energies and thus the TRPES can be simulated. The accuracy of such calculations, however, also depends on the approximations made for the calculation of the ionization transition dipole moments. Most simply, these can be set to unity, which effectively leads to the population of those discretized continuum states that are resonant with the energy of the probe photons. While this allows for the calculation of the coarse shape of the photoelectron spectrum, it still neglects the possible modulation of photoionization cross sections, e.g. by vibrational Franck-Condon factors, the influence of which has been investigated on a model level in Ref. ${ }^{51}$, or by the Koopmans correlations between the involved electronic states of the parent molecule and its ionized counterpart. 
The full information accessible in time-resolved photoionization experiments, however, is not restricted to the energy distribution, but also includes the spatial anisotropy of the photoelectrons. To obtain this quantity, the ionization transition dipole integrals,

$$
\langle\boldsymbol{k}| \otimes\left\langle\Phi^{N-1}|\boldsymbol{E} \cdot \boldsymbol{r}| \Phi^{N}\right\rangle,
$$

have to be calculated accurately, taking into account both the bound electronic state $\left|\Phi^{N}\right\rangle$ of the molecule from which ionization takes place, and the state of the ionized system, which consists of the antisymmetrized product of the bound molecular core $\left|\Phi^{N-1}\right\rangle$ and the free electron scattering state $|\boldsymbol{k}\rangle$. According to the procedure developed by Humeniuk et al. ${ }^{52}$, this can be performed as follows:

In the first place, in the expression (4) the integration over all but one electron coordinate can be performed, giving rise to

$$
\left\langle\boldsymbol{k}|\boldsymbol{E} \cdot \boldsymbol{r}| \phi^{D}\right\rangle,
$$

where

$$
\left|\phi^{D}\right\rangle=\sqrt{N}\left\langle\Phi^{N-1} \mid \Phi^{N}\right\rangle,
$$

is a molecular Dyson orbital. This quantity can be viewed as the one-electron orbital from which the electron is ejected and accounts as well for the reconfiguration of the remaining $\mathrm{N}-1$ electron system after the ionization event, thus including all effects approximately described by Koopmans correlations. 
It can be shown that the differential cross section for photoionization, which determines the spatial and energetic distribution of the photoelectrons, depends on the transition dipole integral according to

$$
\frac{d \sigma}{d \Omega} \propto\left|\left\langle\boldsymbol{k}|\boldsymbol{E} \cdot \boldsymbol{r}| \phi^{D}\right\rangle\right|^{2}
$$

Since the electric field $\boldsymbol{E}$ and the electron momentum $\hbar \boldsymbol{k}$ are defined in the laboratory frame while the Dyson orbital and the scattering wavefunction are defined in the molecular frame, the matrix element can only be calculated after one frame has been rotated into the other. If the differential cross section is subsequently averaged over the initial orientations of the molecules with respect to the laboratory frame, the resulting quantity can be directly linked to the experimental photoionization cross section $\sigma$ and anisotropy $\beta_{2}$ :

$$
\left(\frac{d \sigma}{d \Omega}\right)_{\text {rot. avg. }} \propto \frac{\sigma}{4 \pi}\left[1+\beta_{2} P_{2}(\cos \theta)\right]
$$

In Ref. ${ }^{52}$ as well as in the present contribution, the molecular Dyson orbital is obtained from time-dependent density functional theory by assigning wavefunctions to the $\mathrm{N}$ or $\mathrm{N}-1$ electron molecular states that are constructed as linear combinations of singly excited Slater determinants ${ }^{53}$. The expression (5) then ultimately reduces to overlap integrals between basis functions of the neutral and ionized system. For the ejected photoelectron, in principle the molecular scattering state would need to be determined, which is computationally very demanding. Therefore, following Ref. ${ }^{52}$, the scattering states are approximated by Coulomb functions for the ionization of neutral molecules as considered here. For photodetachment from anions, free plane waves can be employed. 
The above described methodology has been successfully employed to simulate the TRPES and time-resolved photoelectron anisotropy in a variety of closed-shell molecules ${ }^{52,54-56}$. In the present contribution, its applicability to systems with open shell or carbene character will be illustrated.

\section{Resonance-stabilized open-shell species}

Typically, open-shell systems are short-lived species that rapidly react with other molecules in their vicinity. Therefore, they often play a key role as intermediates in complex reaction sequences, such as combustion processes, where they are formed in situ and are consumed by subsequent reaction steps. In the case of combustion, this leads to the formation of polycyclic aromatic hydrocarbons and eventually soot ${ }^{3}$. However, several structural factors can enhance the stability of radicals, such as e.g. steric hindering, which makes the radical center less accessible for reactants. The most striking example of such a stabilizing effect is the triphenylmethyl radical, which is even stable in solution ${ }^{57}$.

Another factor promoting stability is given by resonance of the unpaired electron with adjacent double bonds or aromatic systems. This ultimately leads to the inclusion of the unpaired electron in a delocalized $\pi$-system, thereby lowering the electronic energy and reducing the reactivity. The excited-state dynamics of several such resonance-stabilized open-shell species is discussed in this chapter, including e.g. the allyl and the benzyl radical. Hydrocarbons with a more unusual electronic structure such as the biradicoloid para-xylylene and the carbene cyclopropenylidene are discussed immediately after. Hydrocarbons lacking any $\pi$-orbitals, such as alkyl radicals, do not experience a resonance stabilization and therefore tend to be more reactive. As an example, the dynamics of the tert-butyl radical will be presented in the subsequent chapter 4 .

\subsection{Allyl stabilized radicals}




\subsubsection{Electronic structure and photoreactivity}

The allyl radical is one of the simplest resonance-stabilized hydrocarbons ${ }^{58}$ and an important model for the spectroscopy and dynamics of radicals ${ }^{59}$. It is therefore not surprising that it has aroused considerable interest over the years, which resulted in numerous experimental investigations, employing methods such as $\mathrm{ESR}^{60}, \mathrm{IR}^{61}$, $\operatorname{Raman}^{62-65}$, rotational ${ }^{66}$ and rovibrational spec$\operatorname{troscopy}^{67,68}$, electron diffraction ${ }^{69}$, multiphoton ionization (MPI) ${ }^{70-73,28}$ and photoelectron spectroscopy ${ }^{74-76}$. In parallel, a multitude of theoretical studies has also been performed, covering fundamental aspects such as electron delocalization ${ }^{58}$, spin density distribution ${ }^{77}$ or wavefunction stability $^{78}$ as well as investigating the vibrational spectra ${ }^{79-81}$, excited electronic states ${ }^{82-84}$ and photochemical reactions ${ }^{85}$. Here we will focus on the influence of a methyl group on the excited-state dynamics by comparing the allyl radical with its 1- and 2-methylated counterparts.

The electronic ground state structure of the allyl radical is $\mathrm{C}_{2 \mathrm{v}}$ symmetric ${ }^{84}$, and the position of its excited states as well as those of its derivatives are depicted in Figure 1 (Note that several bands for the allyl radical have been reassigned recently). Exciting into the first bright state, $A{ }^{2} B_{1}(\pi n)$ ${ }^{82,84}$, situated between 3.02-3.35 eV, leads to the loss of a hydrogen atom in the electronic ground state and predominantly to the formation of allene ${ }^{86,87}$. The next three states B-D lie all between 4.96-5.24 $\mathrm{eV}^{73}$. In agreement with the general trends outlined above, the $\mathrm{B}^{2} \mathrm{~A}_{1}$ state already has 3s Rydberg character, and the purely electronic transition $\left(\mathrm{B}^{2} \mathrm{~A}_{1} \leftarrow \mathrm{X}{ }^{2} \mathrm{~A}_{2}\right)$ is one-photon forbid$\operatorname{den}^{28,73,82,84}$. However, several transitions involving vibrational excitation are allowed and have been observed in [1+1] REMPI experiments ${ }^{73,88}$. Upon excitation, dissociation takes place after internal conversion to the ground state, in most instances ejecting a hydrogen atom ${ }^{89,90}$. As a minor channel (16\%), abstraction of a methyl group has been observed as well ${ }^{91}$. The states $\mathrm{C}$ and $\mathrm{D}$ have been assigned as two 3p Rydberg states lying in the molecular plane, which are dark or only weakly 
allowed in one-photon absorption ${ }^{73,82,84}$. The bright $\mathrm{E}\left({ }^{2} \mathrm{~B}_{1}\right)$ state is centered around $5.5 \mathrm{eV}^{73,92,93}$ and has been identified as a 3p Rydberg state perpendicular to the allyl plane ${ }^{73,84}$. Several higherlying Rydberg states, close to the experimental IP of $8.14 \mathrm{eV}^{70}$ have also been reported ${ }^{70}$.

Studies on the electronic states of 1MA and 2MA are much sparser. 1MA exhibits two isomers (E/Z), which according to our density functional theory (DFT) calculations presented in the Supplementary Information (SI) differ in energy by about $0.03 \mathrm{eV}$ (cf. Tables $\mathrm{S} 1$ and S2), with the E isomer being slightly more stable. They have somewhat different $\mathrm{IEs}^{94}$ and different excitation energies as well (cf. our time-dependent DFT (TDDFT) calculations summarized in the SI, Tables S3 and S5).

In Fig. 1, the available experimental (and, where these are lacking, theoretical) data on the lowlying excited states of allyl as well as 1MA and 2MA are summarized. As can be seen, the lowlying excited electronic states of 1MA and 2MA resemble those of the allyl radical: The lowest adiabatic excitation energies (assigned as the A state) have been calculated around $3 \mathrm{eV}$ both for $1 \mathrm{MA}^{95}$ and $2 \mathrm{MA}^{96}$, and have been attributed to a $\pi$ n valence excitation. An experimental value of $2.93 \mathrm{eV}$ has been determined for $1 \mathrm{MA}^{95}$.

In addition, for 2MA, a weak absorption has been observed around $4.75 \mathrm{eV}^{72,92,96,97}$, and has been assigned to a 3s Rydberg state, which is only weakly allowed in one-photon absorption ${ }^{72,92}$. At higher energies, a strong band is observed around 5.1-5.4 $\mathrm{eV}^{92,96-98}$, which is identified as a bright $3 p$ Rydberg state ${ }^{92,93}$, similar to the case of allyl, albeit slightly red-shifted. A similar bright band has been found for 1MA between 5.2-5.4 $\mathrm{eV}^{97}$.

In an attempt to complement the previous experimental and theoretical findings, we have investigated the excited electronic states of $1 \mathrm{MA}$ and 2MA employing different quantum chemical 
methods. In Ref. ${ }^{93}$ we provide benchmark data on $2 \mathrm{MA}$, confirming the experimentally established sequence of states $\pi \mathrm{n}, 3 \mathrm{~s}$, bright $3 \mathrm{p}$. At slightly higher energies, we found the two remaining and almost dark Rydberg 3p states. For the TDDFT methodology chosen for the simulation of the nonadiabatic relaxation dynamics presented in Section 3.1 .2 below (CAM-B3LYP ${ }^{99} / 6$ $\left.31++\mathrm{G}^{100,101}\right)$, the energetics of the lowest excited states of $2 \mathrm{MA}$ and their characterization in terms of natural transition orbitals (NTOs) ${ }^{102}$ are also provided in the SI, Fig. S2 and Table S6.

The excited state energies of 1MA obtained using different TDDFT and EOM-CCSD ${ }^{103,104}$ quantum chemical models are presented in Tables S3 ( $\mathrm{E}$ isomer) and S5 ( $\mathrm{Z}$ isomer). The state character for the E isomer is illustrated using NTOs in Table S4 and Fig. S1. From our computations it can be concluded that the experimentally observed bright absorption in the range of 5.2$5.4 \mathrm{eV}$ can be attributed to two states exhibiting both valence-excited $\pi \pi^{*}$ and $3 p$ Rydberg character. The involved Rydberg orbital is perpendicular to the allyl plane, completely analogous to what was previously established for allyl and 2MA. Between this set of states and the low-lying A $(\pi n)$ state, a 3s Rydberg state as well as the two other components of the 3p Rydberg state are found computationally, which are all almost dark in one-photon absorption. It should be noted that the ordering of states in $1 \mathrm{MA}$ is distinctly different to the one found for 2MA: While in 2MA the bright 3p Rydberg state immediately follows the 3s state, and the dark 3p Rydberg states lie higher (cf. also SI, Table S6), the situation is reversed for 1MA, where the dark 3p Rydberg states lie between the $3 \mathrm{~s}$ state and the bright states.

Regarding photochemical reaction pathways, exciting into the A-state of 1MA also leads to the loss of a hydrogen atom ${ }^{95}$ just as for the allyl radical. For the $2 \mathrm{MA}$, as discussed above, the A state has not yet been experimentally observed. For excitation of $2 \mathrm{MA}$ into the band around $4.75 \mathrm{eV}$, loss of a hydrogen atom is found as well ${ }^{105}$, similar to the B-state in the allyl radical. 


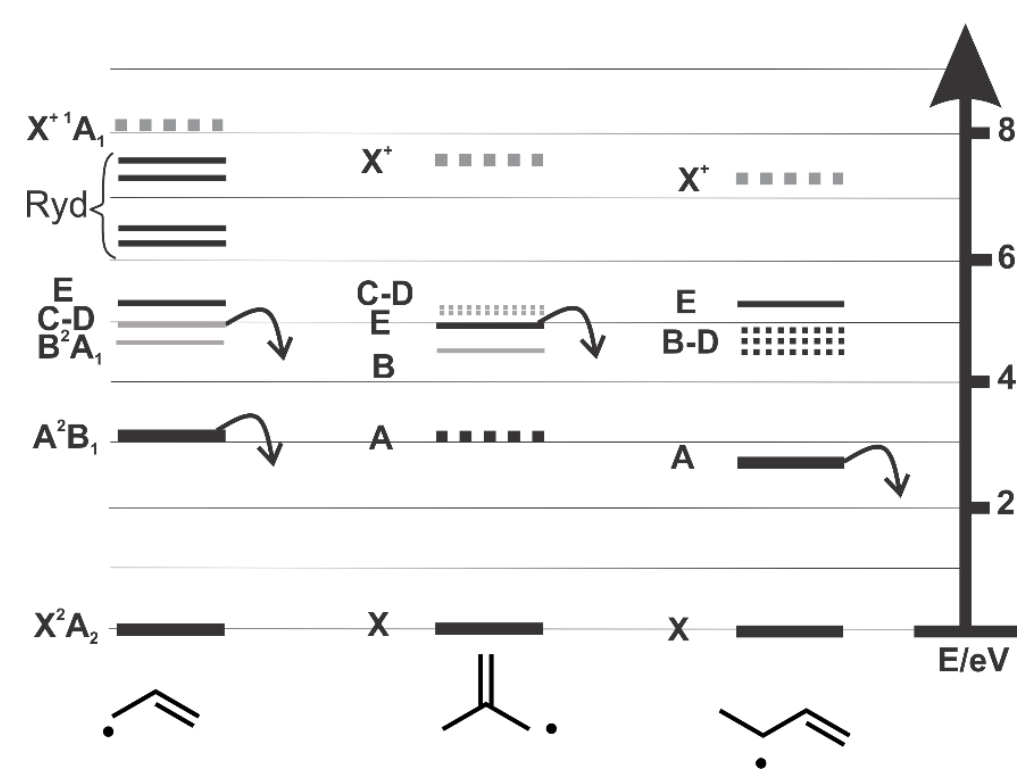

Figure 1. Overview of the electronic states for the allyl radical (left), 2MA (middle) and 1MA (right) relative to their respective ground state. Thick black lines represent one-photon excitable states, grey thick lines represent transitions that are one-photon forbidden. Rydberg states are drawn in as thinner black lines, whereas cationic states are represented as grey, dotted lines. Dotted lines for the neutral species correspond to theoretically predicted dark states. States from which photodissociation has been observed (either ejection of a hydrogen atom or a methyl group), are marked with an arrow. For the correspondence between experimental and theoretical excited state assignments cf. SI, Table S7. For references see text.

Comparing the IEs, the value for the allyl radical $\left(8.1309 \mathrm{eV}^{106}\right)$ is as expected about $1.60 \mathrm{eV}$ lower than the one of propene $\left(\mathrm{IE}=9.73 \mathrm{eV}^{107}\right)$. The additional methyl group lowers the IE once more by at least $0.3 \mathrm{eV}(2 \mathrm{MA}: 7.88 \mathrm{eV}, E-1 \mathrm{MA}: 7.48 \mathrm{eV}, Z-1 \mathrm{MA}: 7.59 \mathrm{eV})^{94}$.

\subsubsection{Relaxation dynamics}

To better understand the coupling between the excited states in these three open-shell hydrocarbons, their dynamics has been studied using a combination of TRPES and theory. The allyl radical 
was the first hydrocarbon radical examined by TRPES ${ }^{108-110}$ using a magnetic bottle setup. The Bstate was excited with two photons into the origin $0_{0}^{0}$ and the $7_{0}^{1}$ fundamental, corresponding to the CCC bending mode ${ }^{108}$ and a mono-exponential decay was observed in both cases. The timeconstant decreased from 22 to 16 ps with the vibrational excitation energy increasing by $\sim 0.05 \mathrm{eV}$. Data for vibrational overtones confirmed this trend ${ }^{110}$ which is expected for most possible deactivation pathways. Since the molecule does not possess low lying electronic states of higher spin multiplicity ${ }^{84}$ and intersystem crossing can thus be ruled out, predissociation and internal conversion (IC) remain as possible deactivation pathways. Loss of a hydrogen atom has been observed from the electronic ground state on a ns-time-scale ${ }^{89}$, leaving IC as the deactivation pathway on the ps time scale. With only the time-constant of the first deactivation step however, it is not possible to determine whether the B-state relaxes to the A-state or directly to the ground state ${ }^{108}$.

Overall, increased excitation is accompanied by decreasing time constants, e.g. from 20 ps for the B $7_{0}^{1} 12_{0}^{1}$ state to $13 \mathrm{ps}$ for the B $16_{0}^{1}$ state $(+0.09 \mathrm{eV})$ and from $12 \mathrm{ps}$ for the $\mathrm{C} 0_{0}^{0}$ state to 9 ps for the $\mathrm{C} 7_{0}^{1}$ state $(+0.05 \mathrm{eV})^{110}$.

To clarify whether or not the ground state is reached directly from the higher excited states, the effect of deuteration on the dynamics was also investigated ${ }^{109}$. Deuteration increases the vibrational density of states while the Franck-Condon factors between the involved vibronic states decrease. If the B-state relaxes directly to the ground state, the dynamics should be governed by its density of states, thus the time-constants should be unchanged or even increase upon deuteration. In the case of an internal conversion to the A-state, which is governed by the magnitude of the Franck-Condon factors, we should see a slower decay. Indeed, upon deuteration of the allyl radical the excited-state lifetime of the $\mathrm{B} 70^{1} 12_{0}{ }^{1}$ (earlier assigned as $\mathrm{C} 7_{0}^{1}$-state) increased drastically from 22 to $89 \mathrm{ps}$, which is consistent with internal conversion to the A-state ${ }^{109}$. The A-state itself decays 
faster than the resolution of the ps-setup used in the experiment ${ }^{109}$, in agreement with the broad UV absorption bands of the A-state ${ }^{111}$.

If we now substitute a hydrogen atom with a methyl group, we increase not only the vibrational density of states by introducing new modes, but for specific normal modes the vibrational periods will strongly change due to the higher moment of inertia associated with a methyl group instead of a hydrogen. This means that molecular motions involving these normal modes will proceed more slowly, which in turn influences the excited-state dynamics. The term 'inertial effect' has been coined for the promotion of some deactivation pathways over others via the modification of specific vibrational motions due to substituents ${ }^{11,112-114}$. Before turning to the TRPES of 2MA/1MA, it should be noted that the excited-state dynamics of the B state of $2 \mathrm{MA}$ has been examined using $\left[2+1^{\prime}\right]$ ps-time resolved mass spectrometry ${ }^{115}$, and it has been found that the vibronic levels in the B-state of 2MA have a significantly shorter life-time (30-50\%) than equally excited states of the allyl radical ${ }^{111}$.

The TRPES of the two methyl allyl radicals has been measured after excitation to the intense absorption bands around $5.2 \mathrm{eV}$ and is presented in Figure 2. In the left part, the data of 2MA published in Ref. ${ }^{93}$ are shown, while on the right, we present previously unpublished results for a mixture of $(Z / E)$-1MA, obtained using the same experimental setup as described in Ref. ${ }^{93}$. 

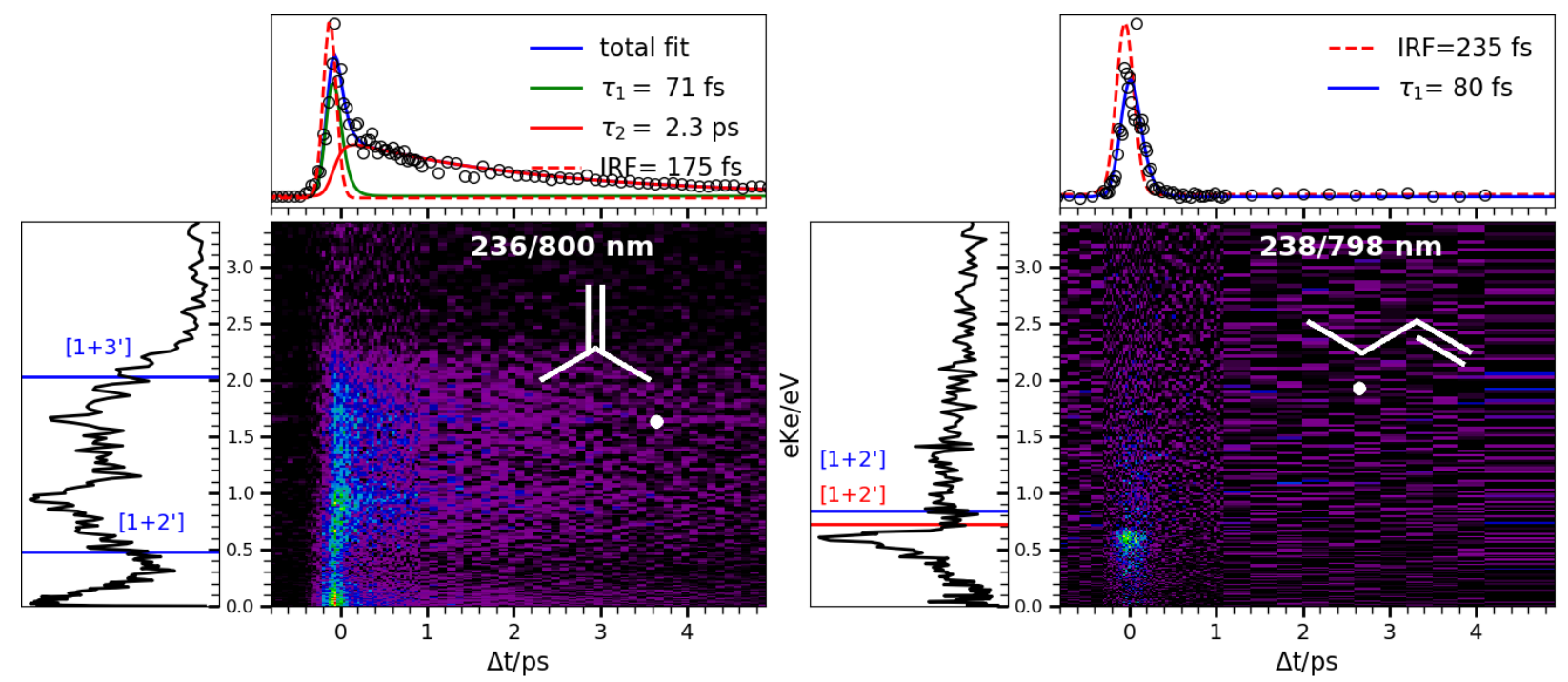

Figure 2. TRPES of the 2MA (left) and 1MA (right) radical. The upper panels show the total photoelectron decay (experimental points: open black circles), fitted with either a sequential biexponential fit (left) or a monoexponential fit (right). IRF is the instrument response function. The left panel shows the total photoelectron spectrum, summed over all measured times with relevant signal. Ionization limits are indicated in blue/red, with $\operatorname{IE}(2 \mathrm{MA})=7.88 \mathrm{eV}, \operatorname{IE}(\mathrm{E}-1 \mathrm{MA})=7.48 \mathrm{eV}$ and $\operatorname{IE}(\mathrm{Z}-1 \mathrm{MA})=7.59 \mathrm{eV}^{94}$.

In the case of $2 \mathrm{MA}$ we observe a fast sequential biexponential decay with $\tau_{1}=208 \mathrm{fs}$ and $\tau_{2}=4.1$ ps for a pump wavelength of $240.6 \mathrm{~nm}$, which shortens to $\tau_{1}=71$ fs and $\tau_{2}=2.3$ ps for a pump wavelength of $236 \mathrm{~nm}$ (top panel in Figure 2, left). A change in the photoelectron spectrum in association with these two different time-constants was not observed, but since $\tau_{1}<\mathrm{IRF}$, the $\tau_{1^{-}}$associated signal is 'drowned' in the $\tau_{2}$-associated signal. The state corresponding to $\tau_{2}$ is clearly identifiable in the photoelectron spectrum (side panel in Figure 2, left): the signal stems from ionization by up to three probe photons (indicated as blue lines), and the peaks between 0.65 and 1.4 $\mathrm{eV}$ are attributed to intermediate resonances, from which ionization finally takes place. Using multiphoton schemes such intermediate resonances have been observed before ${ }^{116-118}$, but these are not 
observable with one-photon probes as used for the allyl radical. Regarding the nature of the intermediate resonance, evidence hints at s-type Rydberg states, which can be reached from the initially excited 3p Rydberg state $\left(D_{3}\right)$ in a two-photon transition $(\Delta n= \pm 1, s \rightarrow p \rightarrow s(d))$. This is supported by the photoelectron anisotropy showing a $\beta_{2}$ value near 2 , which is characteristic for ionization from an s state ${ }^{1}$. Just like for the allyl radical, the overall mechanism is a stepwise internal conversion from the initially excited state to the ground state ${ }^{93,108-110}$.

${ }^{1}$ The ionization potential of a Rydberg state, $I E_{R y d b e r g}=\frac{R y}{(n-\delta)^{2}}$, can be related to the photoelectron kinetic energy eKe by $I E_{R y d b e r g}=h v-e K e$ (where Ry is the Rydberg constant, $\mathrm{n}$ the principal quantum number, $\delta$ the quantum defect and $h v$ the photon energy). Assuming ionization by one $1.55 \mathrm{eV}$ photon, the peaks in the spectrum correspond to $\delta=1$ and $\mathrm{n}=5 / \mathrm{n}=7$. 

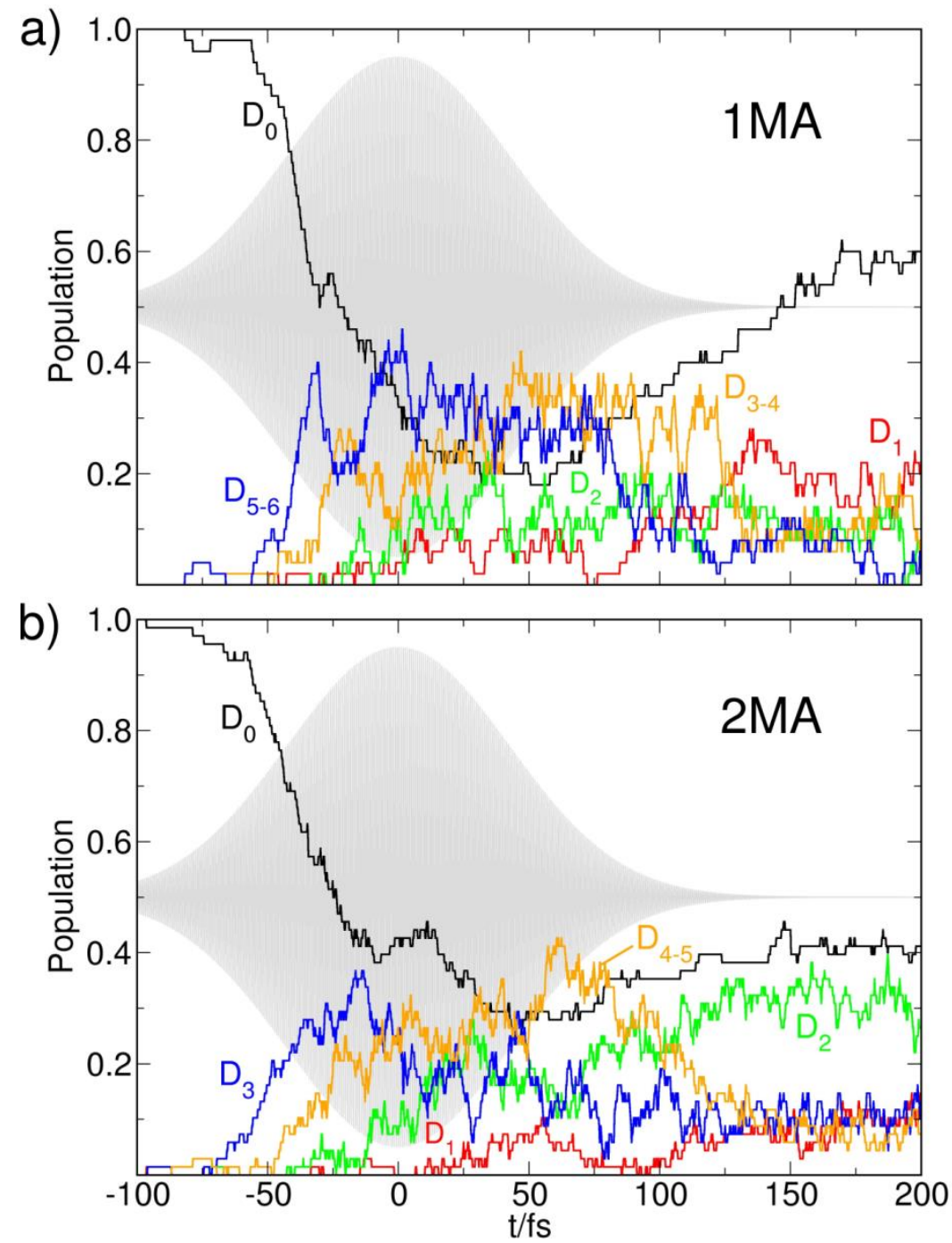

Figure 3. Time-dependent electronic state populations of (a) 1MA (E isomer) and (b) 2MA induced by a Gaussian laser pulse resonant to the bright transition around $5.4 \mathrm{eV}$. The blue lines correspond initially to the bright states of mixed Rydberg $3 \mathrm{p}_{\mathrm{y}}$ and $\pi \pi^{*}$ character in $1 \mathrm{MA}$, or to the single bright $3 p_{\mathrm{y}}$ Rydberg state in $2 \mathrm{MA}$, the orange lines indicate the two dark Rydberg $3 p$ states. The laser pulse envelope is shown in grey in the background. The populations are averaged over 50 trajectories for $1 \mathrm{MA}$ and 70 trajectories for $2 \mathrm{MA}$.

For a deeper insight into the molecular mechanism of the relaxation dynamics of $2 \mathrm{MA}^{93}$ we simulated this process using the $\mathrm{FISH}^{42}$ approach. To this end, an ensemble of 70 initial conditions 
was sampled from a $10 \mathrm{ps}$ ground state classical trajectory propagated at a constant temperature of $200 \mathrm{~K}$. The FISH trajectories were then propagated under the influence of a $100 \mathrm{fs}$ Gaussian laser pulse resonant to the brightest transition below $5.5 \mathrm{eV}$, which corresponds to the $\mathrm{D}_{0} \rightarrow \mathrm{D}_{3}\left(3 \mathrm{p}_{\mathrm{y}}\right.$ Rydberg, perpendicular to the molecular plane ${ }^{2}$ ) transition. The nonradiative deactivation after the end of the pulse was followed for about $300 \mathrm{fs}$. The quantum chemical method of choice in this case was TDDFT employing the CAM-B3LYP functional ${ }^{99}$ and the $6-31++\mathrm{G}$ basis set ${ }^{100,101}$. As discussed in detail in Ref. ${ }^{93}$, this methodology represents a good compromise between accuracy and computational efficiency to be employed for the FISH simulation of 2MA. The resulting electronic state population dynamics are displayed in Figure 3.

The laser pulse initially excites the ensemble of molecules to the third excited state $\mathrm{D}_{3}$, which has $3 p_{y}-$ Rydberg character. This state couples with $D_{4}\left(3 p_{x}\right.$ Rydberg) and $D_{5}\left(3 p_{z}-R y d b e r g\right)$, which are nearly degenerate with $\mathrm{D}_{3}$ but possess far lower oscillator strength. The population then rapidly flows to the $\mathrm{D}_{2}(3 \mathrm{~s})$ state $^{92},{ }^{93}$ in agreement with the analysis of the photoelectron spectra discussed above. Finally, on a ps time scale the ground state is reached, with the intermediate $\mathrm{D}_{1}(\pi \mathrm{n})$ state being only transiently populated due to rapid passage through conical intersections. After this, the electronic excitation is fully converted to vibrational energy. This allows the radical to overcome the barrier for fragmentation to release a hydrogen atom. Such hydrogen loss following excitation into the p-Rydberg state has been reported before ${ }^{105}$, and has been reproduced in the simulation.

\footnotetext{
${ }^{2}$ Note that we adopt here the coordinate axis definitions of Ref. [77], such that the allyl $\mathrm{C}_{2}$ axis corresponds to the z-axis, and the y-axis is perpendicular to the molecular plane.
} 
Overall, the simulations reveal a time scale of about $300 \mathrm{fs}$ for relaxation from the initially excited states to $D_{1}$, which is in full agreement with the experimental findings for the decay of the photoelectron signal.
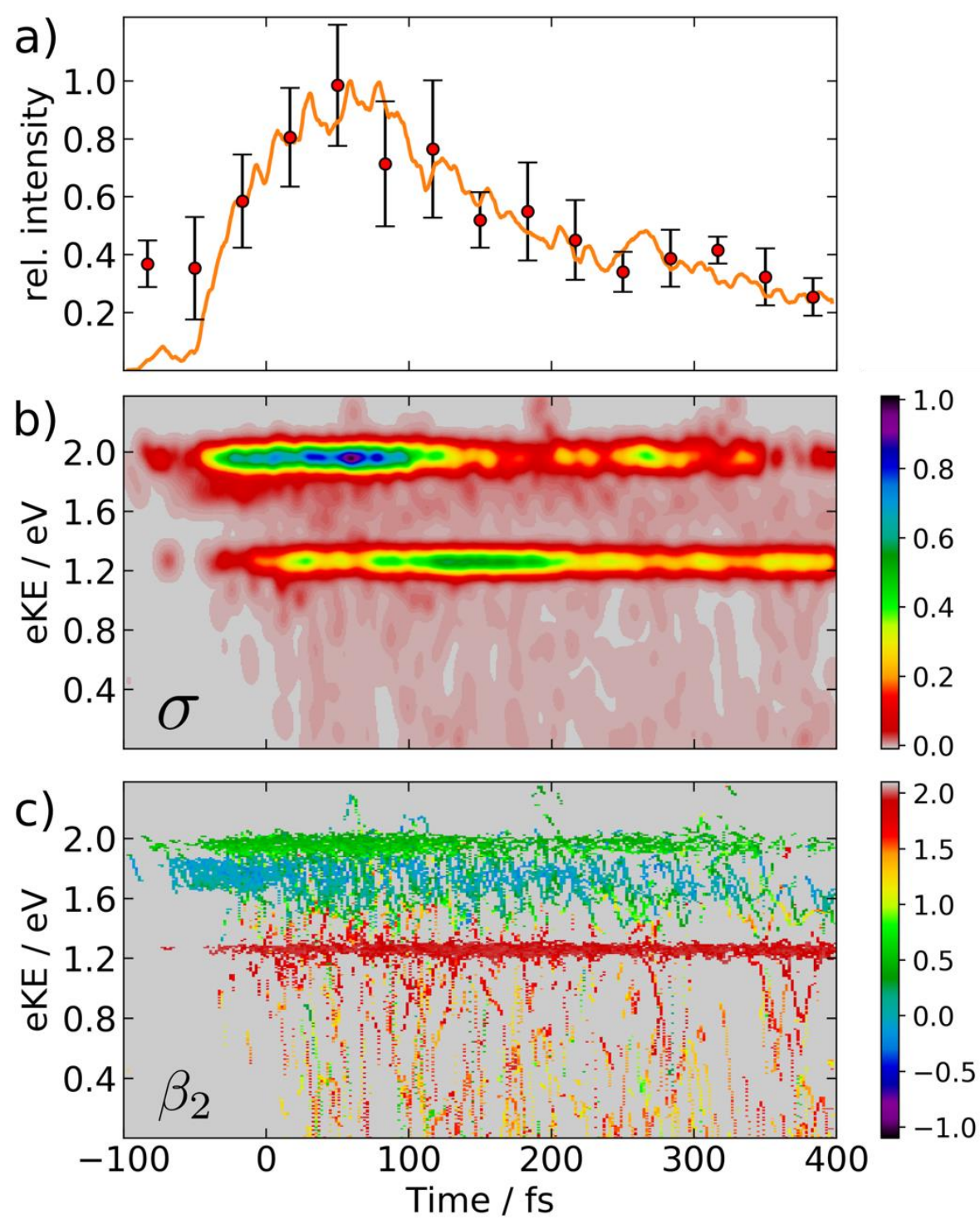

Figure 4. (a) Experimental (red dots) and simulated (orange line) time-dependent photoelectron intensities of 2MA, (b) simulated TRPES for $266 \mathrm{~nm}$ one-photon ionization, (c) simulated timeresolved photoelectron anisotropy parameter $\beta_{2}$ corresponding to the TRPES shown in (b). 
Based on the FISH simulation, for 2MA we additionally calculated the TRPES and the corresponding PAD parameter $\beta_{2}$, employing the approach outlined in the Methods section (cf. also Ref. ${ }^{52}$ ). These quantities correspond to a one-photon ionization process, thus the specific energy and anisotropy distribution differ from the experimental data. Nevertheless, the energy-integrated photoelectron intensity obtained as a function of time agrees well with the experimental time traces, as presented in Figure 4. Taking the opportunity to provide a theoretical reference for future one-photon-ionization experiments, we present the simulated TRPES and time-resolved photoelectron anisotropies in Figure 4b/c. It can be seen from Figure $4 \mathrm{~b}$ that the signal at $2 \mathrm{eV}$, corresponding to the initially excited manifold of $3 p$ Rydberg states, maximizes around $150 \mathrm{fs}$ and then rapidly fades away on a time scale of 300-400 fs, while a lower energy signal at $1.3 \mathrm{eV}$ appears time-delayed, maximizing around $250 \mathrm{fs}$ and decreasing slowly within the simulation time of 500 fs. Inspection of the time-resolved anisotropies presented in Figure $4 \mathrm{c}$ makes clear that the signal at $1.3 \mathrm{eV}$, which exhibits a high photoelectron anisotropy of about 2, corresponds to the $3 \mathrm{~s}$ Rydberg state $\left(\mathrm{D}_{2}\right)$ intermediately populated in the course of the relaxation process. The 3p-Rydberg states, by contrast, feature much smaller anisotropies around a value of zero. This illustrates nicely that in specific cases the photoelectron anisotropy can provide a versatile means to distinguish electronic states of different character.

In contrast to the rich dynamics observed in 2MA, the TRPES of 1MA after excitation to the bright absorption band around $5.2 \mathrm{eV}$ (see Figure 2, right) only shows ionization to the cationic ground state which disappears in less than $80 \mathrm{fs}\left(\tau_{1}\right)$. This time-constant can either represent the fast depopulation of the initially excited state, e.g. via passage through a conical intersection, the change in Franck-Condon factors as the wavepacket moves out of the observational window, or a 
direct dissociation. Our theoretical simulations discussed below indicate that indeed a rapid depopulation of the initially excited state takes place, which is, according to the experimental data, faster than for $2 \mathrm{MA}$ by roughly a factor of 3 . This indicates that not only the energies are strongly influenced by the geometric position of the methyl group, but also the dynamical couplings between the excited states, as has been observed in closed-shell molecules before ${ }^{112,119}$. The fact that we do not observe intermediate Rydberg states for 1MA as we did in the case of the $2 \mathrm{MA}$ is an additional indication for a fast deactivation to lower-lying states.

To gain a detailed picture of the relaxation processes following initial excitation, we simulated the light-induced dynamics using the FISH method in the same way as for 2MA (see above). Specifically, we generated an ensemble of 50 initial conditions sampled from a $200 \mathrm{~K}$ classical ground state trajectory of the $\mathrm{E}$ isomer of $1 \mathrm{MA}$. The $\mathrm{Z}$ isomer lies $0.031 \mathrm{eV}$ higher in energy (cf. SI, Tables $\mathrm{S} 1, \mathrm{~S} 2)$, corresponding to a thermal population ratio between the $\mathrm{Z}$ and $\mathrm{E}$ isomers of 0.16 at 200 $\mathrm{K}$. Therefore, we focused our investigation on the dynamics of the E isomer. The FISH trajectories pertaining to the $\mathrm{E}$ isomer were propagated under the influence of a $100 \mathrm{fs}$ Gaussian laser pulse resonant to the bright transition around $5.4 \mathrm{eV}$, which dominantly involves two states of mixed $3 \mathrm{p}$ Rydberg and valence $\pi \pi^{*}$ character $\left(\mathrm{D}_{5}\right.$ and $\left.\mathrm{D}_{6}\right)$, as discussed above. After the pulse had ended, the dynamics was followed for additional $100 \mathrm{fs}$. The resulting electronic state populations are presented in Figure 3a. The laser pulse initially excites the two bright states $\mathrm{D}_{5}$ and $\mathrm{D}_{6}$, from which the population is rapidly transferred via the dark $3 p$ Rydberg states $\left(D_{3} / D_{4}\right)$ and the 3 s Rydberg state $\left(D_{2}\right)$ to the lowest excited state $D_{1}$. Repopulation of the ground state proceeds fast and mostly from the $\mathrm{D}_{1}$ state. $\mathrm{At} \mathrm{t}=200 \mathrm{fs}$, about half of the excited population has returned to the ground state. This rapid decay of excited state population is consistent with the similarly fast disappearance of the experimental photoelectron signal (cf. Figure 2). Notably, the return to the ground state is much 
faster for $1 \mathrm{MA}$ as compared to $2 \mathrm{MA}$. This is possibly caused mainly by the more efficient population of the bright electronic states due to the pump pulse, which is, according to our calculations, accompanied by a stronger increase of the internal vibrational energy of 1MA as compared to 2MA (cf. SI, Fig. S3). As a consequence, the dynamical nonadiabatic coupling $\boldsymbol{d}_{i j}[\boldsymbol{R}(t)] \cdot \dot{\boldsymbol{R}}$ (cf. Eq. (2)), which governs the electronic relaxation after the end of the pulse, increases due to the higher nuclear velocities $\dot{\boldsymbol{R}}$, although the matrix elements $\boldsymbol{d}_{i j}$ are very similar for the two molecules.

\subsection{The Tropyl, Benzyl and related radicals}

In allyl-like systems the resonance of the radical center with one double bond as well as the site of the methyl group influence the excited-state dynamics. How is this situation for larger resonance-stabilized, or even aromatic systems? In the following we will first review our work on the dynamics of the benzyl radical ${ }^{120}$ and present new results for the tropyl radical, before providing a comparison to recently published work on the xylyl radicals ${ }^{121}$.

\subsubsection{Electronic structure}

As the primary product of toluene decomposition, the benzyl radical is a key player in combustion processes and in the formation of soot ${ }^{122-127}$. The tropyl radical, a $\mathrm{C}_{7} \mathrm{H}_{7}$ isomer, is also resonance-stabilized, but its properties, reactivity and spectroscopy are significantly different (see Figure 5). The $\mathrm{C}_{2 \mathrm{v}}$ symmetric benzyl radical is planar, and its unpaired electron interacts with the aromatic ring ${ }^{128}$. The tropyl radical, by contrast, is a non-aromatic ring system. For the aromatic tropylium cation, a highly symmetric heptagonal structure ( $\mathrm{D}_{7 \mathrm{~h}}$ point group) has been established ${ }^{129}$. The additional electron leading to the formation of the neutral radical will, according to theoretical considerations, be placed in a degenerate pair of empty $E_{2}^{\prime \prime}$ symmetric orbitals ${ }^{130}$. Such a degenerate electronic ground state configuration is unstable, leading to a reduction of symmetry 
by geometric distortion (Jahn-Teller effect), thereby lifting the degeneracy ${ }^{131}$. For the tropyl radical the point group is reduced to $\mathrm{C}_{2 \mathrm{v}}$, and the electronic ground state exhibits ${ }^{2} \mathrm{~B}_{1}$ symmetry ${ }^{132,133}$, as has been recently confirmed by Miller et al. using fluorescence spectroscopy ${ }^{134,135}$ as well as by measuring the $\mathrm{C}-\mathrm{H}$ stretch vibrations in helium nanodroplets ${ }^{136}$. However, the energy splitting due to the Jahn-Teller effect is rather small, such that the vibrational zero-point energy may be sufficient to surmount the barriers between several minima of $\mathrm{C}_{2 \mathrm{v}}$ symmetry. This leads to a fluxional geometry of tropyl, and the seven protons of the molecule appear to be equivalent in ESR spectroscopy ${ }^{137}$. Thus the Jahn-Teller effect has to be taken into account to properly describe the vibrational band structure which is spectroscopically observed.

The different resonance stability of the two molecules is also reflected in their heats of formation, with benzyl being about $44.4 \mathrm{~kJ} / \mathrm{mol}$ more stable than tropyl ${ }^{138,139}{ }^{3}$. Once ionized, this situation is reversed: now the tropyl cation is aromatic ( 6 electrons in a ring) and more stable by about 54.8 $\mathrm{kJ} / \mathrm{mol}$ than the benzyl cation ${ }^{138,140}$, which implies a lower IE of $6.23 \mathrm{eV}^{141}$ compared to $7.252 \mathrm{eV}$ for the benzyl radical ${ }^{142}$.

The excited states of the benzyl radical have been examined in several studies. Strong fluorescence has been observed between $400-600 \mathrm{~nm}$ and attributed to the lowest excited $\mathrm{A}^{2} \mathrm{~B}_{2}$-state ${ }^{143-}$ ${ }^{147}$, which couples strongly to the nearly isoenergetic $\mathrm{B}^{2} \mathrm{~A}_{2}$-state ${ }^{148}$, whose symmetries have been reassigned recently ${ }^{120}$. These two states have been confirmed by various theoretical approaches to be of valence $\pi \pi^{*}$ character ${ }^{120,149,150}$. The next higher $\mathrm{C}$ state lies at $4.06 \mathrm{eV}^{151} 152$ and was recently reassigned to a Rydberg-type ${ }^{2} \mathrm{~A}_{1}$ (3s) state ${ }^{120}$. Between 4 and $5.6 \mathrm{eV}$, the strongly absorbing $\mathrm{D}$

${ }^{3}$ However, the tropyl radical is still relatively strongly resonance-stabilized, even better than the triphenylmethyl radical ${ }^{199}$. 
band is observed. ${ }^{153}$. Only recently, it could be shown that this band is actually comprised both of valence $\pi \pi^{*}$ states as well as of p-type Rydberg states, with the highest oscillator strength being exhibited by an $\mathrm{A}_{2}$-symmetric $\pi \pi *$ state and a close-lying Rydberg p-state of $\mathrm{B}_{2}$ symmetry ${ }^{120}$.

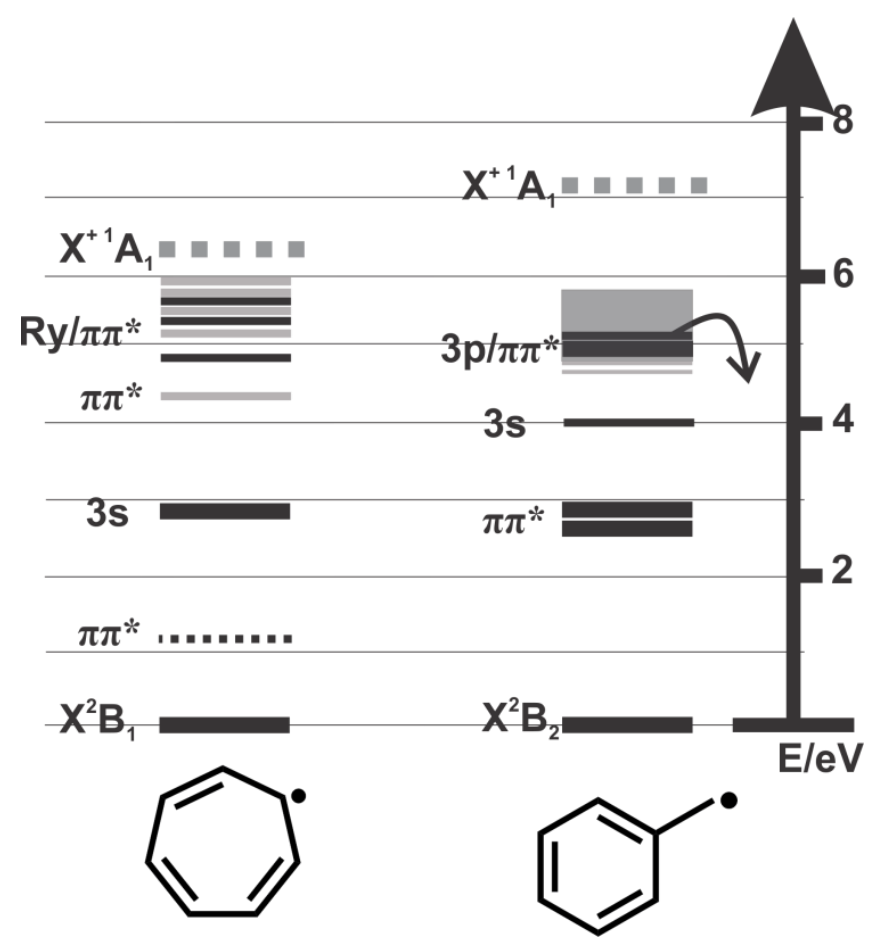

Figure 5. Overview of the electronic states for the tropyl radical (left) and benzyl radical (right) relative to their respective ground state. Thick black lines represent one-photon excitable states, grey thick lines represent transitions that are one-photon forbidden. Rydberg states are drawn in as thinner black lines, whereas cationic states are represented as grey, dotted lines. Dotted lines for the neutral species correspond to theoretically predicted dark states. States from which photodissociation has been observed (either ejection of a hydrogen atom or a methyl group), are marked with an arrow. The gray box corresponds to the several theoretically determined electronic states in that region. For references see text. 
The lowest-lying excited state (A) of the tropyl radical has been investigated by laser-induced fluorescence ${ }^{135}$ as well as REMPI spectroscopy ${ }^{154}$, revealing a large vibronic progression in the energy range between 3.2-3.9 eV. This state is considered to have ${ }^{2} \mathrm{E}_{2}$ " symmetry in a $\mathrm{D}_{7 \mathrm{~h}}$ geometry, but the large vibronic progression of both the fluorescence and the excitation spectra, covering over $6000 \mathrm{~cm}^{-1}$, indicate Jahn-Teller distortion ${ }^{135,154}$. Several higher-lying electronic transitions have been observed by one-photon absorption ${ }^{155}$ and two-photon REMPI ${ }^{132}$ in the range between 4.3-4.7 eV as well as between 5.2-6.0 eV, and have been assigned as Rydberg states.

Calculations for the tropyl radical are challenging, not the least by the occurrence of the JahnTeller distortion, which already complicates the ground state geometry optimization. We have performed a set of benchmark ab initio calculations of the excited electronic states, the details of which are given in the SI, Part E. Briefly, based on the $\mathrm{C}_{2 v}$-symmetric electronic ground state structure shown in Fig. S4 (with Cartesian coordinates provided in Table S8), we have calculated the excitation energies employing the EOM-CCSD ${ }^{103,104}$ and CAS-MRCI ${ }^{156-158}$ methods. Due to the ubiquitous presence of diffuse Rydberg states, the calculations have been performed using basis sets augmented by up to three sets of diffuse functions. The results summarized in Table S9 make clear that, compared to benzyl, an even larger number of energetically close-lying excited states is present in the energy range up to $5 \mathrm{eV}$ relevant for the TRPES measurements discussed below. These include several $\pi \pi^{*}$ valence excited states as well as a complete series of $3 \mathrm{~s}, 3 \mathrm{p}$ and 3d Rydberg states. Beyond $\sim 4.6 \mathrm{eV}$, the lowest states of the $\mathrm{n}=4$ Rydberg series are also found computationally, although the precise position of these states cannot be unambiguously determined.

\subsubsection{Relaxation dynamics}


The A-state of benzyl is long-lived and fluorescent ${ }^{143-147}$, while the C-state already shows a fast dynamics ${ }^{152}$. After excitation a biexponential decay was seen in the time-resolved mass spectra, with $\tau_{1}=400$ fs and $\tau_{2}=4.5$ ps for the $0^{0}$ band. Both time-constants shorten to 180 fs and $2.1 \mathrm{ps,}$ respectively, when the $4^{1}$ band is excited. The biexponential decay was interpreted as IC from the initially excited $\mathrm{C}$ state to the $\mathrm{A} / \mathrm{B}$-state with another IC to the ground state, and the decreasing lifetime as a reflection of the increasing density of vibrational states in the relevant energy region, in agreement with Fermi's Golden Rule. In an earlier experiment ${ }^{159}$, using time-resolved mass spectroscopy, the D-state was excited at $255 \mathrm{~nm}(4.86 \mathrm{eV})$, and a short-lived transient ( $\left.\tau_{1}=150 \mathrm{fs}\right)$ was observed, decaying to a constant signal level. This offset was interpreted as a residual from a lower-excited state or ground state, consistent with elimination of a hydrogen atom upon D-state excitation ${ }^{160}$, leading to fulvenallene formation from the ground state after internal conversion ${ }^{160}$. We re-examined the D-state of the benzyl radical using TRPES with the goal of unravelling the details of the nonradiative deactivation process ${ }^{120}$.
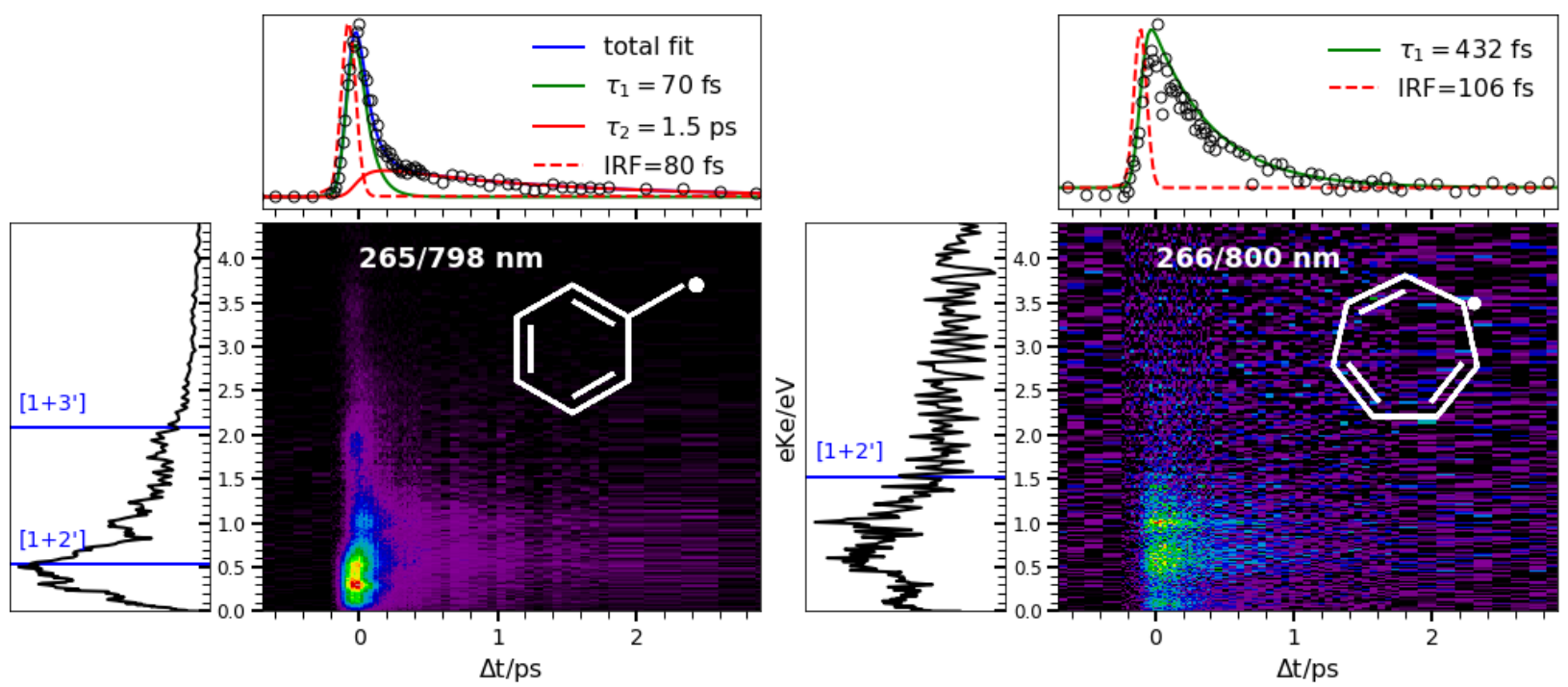

Figure 6. TRPES of the benzyl radical (left) and tropyl radical (right). The upper panels show the total photoelectron decay (experimental points: open black circles), fitted with either 
a sequential biexponential fit (left) or a monoexponential fit (right). IRF is the width of the instrument response function. The left panel shows the total photoelectron spectrum, summed over all measured times with relevant signal. Ionization limits are indicated in blue, with $\mathrm{IE}($ benzyl radical $)=7.252 \mathrm{eV}^{142}$ and $\mathrm{IE}($ tropyl radical $)=6.23 \mathrm{eV}^{141}$.

Benzyl was excited at $265 \mathrm{~nm}(4.68 \mathrm{eV})$ and two different probe schemes were used, multiphoton ionization at $798 \mathrm{~nm}$ (see Figure 6, left) and one-photon ionization at $398 \mathrm{~nm}$ (cf. Ref. ${ }^{120}$ ). The TRPES at the two different probe wavelengths reveal different aspects of the dynamics: With $798 \mathrm{~nm}$ signals from ionization by up to three photons appeared. A structured spectrum and a biexponential decay ( $\tau_{1}=70 \mathrm{fs}, \tau_{2}=1.5 \mathrm{ps}$ ) of the total photoelectron signal were observed. With $398 \mathrm{~nm}$ only one peak at $0.3 \mathrm{eV}$ due to a [1+1']-process was visible, showing a mono-exponential decay ( $\tau_{1}=82 \mathrm{fs}$ ) and corresponding to the ionization into the ionic ground state. The additional peaks in the $[1+3$ '] experiment are caused by intermediate Rydberg state resonances in the probe step, similar to the observations made for 2-MA. For $798 \mathrm{~nm}$ probe the photoelectron spectrum changes over time (see ref. ${ }^{120}$, fig. 7), which indicates that the short time-constant of 70-82 fs observed in the TRPES with both 798 and $398 \mathrm{~nm}$ probe wavelength corresponds to a change in electronic character.

To attribute this change to specific excited states of the benzyl radical, we simulated the nonadiabatic relaxation dynamics employing the surface hopping approach outlined in the Computational section. According to ab initio calculations employing the complete active space self-consistent field method (CASSCF) combined with multireference configuration interaction (MRCI $)^{120}, 3 \mathrm{p}$ Rydberg states constitute a major part of the bright D-band, while a bright $\pi$-type valence-excited state is also present. The lower-lying C state was found to be of Rydberg 3s character. The nonadiabatic relaxation was simulated using the surface hopping method ${ }^{161}$ combined with the CASSCF 
approach, where the seven $\pi$ orbitals and a Rydberg 3s orbital were included in the active space. The dynamics was started in the bright fourth excited state $\left(\mathrm{D}_{4}\right)$, which is the $2{ }^{2} \mathrm{~A}_{2}\left(\pi \pi^{*}\right)$ state. The ensuing time evolution of the diabatized populations of the four lowest excited states is presented in Figure 7. The initially populated state, corresponding to the experimental D band, decays in about $100 \pm 50$ fs to the s-Rydberg state (C band), from which it relaxes to the nearly degenerate, long-lived valence states that constitute the A and B bands within $700 \pm 100 \mathrm{fs}$. We therefore attribute the time-constant in the $398 \mathrm{~nm}$ TRPES and $\tau_{1}$ in the $798 \mathrm{~nm}$ TRPES to this first relaxation from the $\mathrm{D}$ to the $\mathrm{C}$ band, and $\tau_{2}$ in the $798 \mathrm{~nm}$ TRPES to the relaxation of $\mathrm{C}$ to the $\mathrm{A} / \mathrm{B}$ states. Even though we could not experimentally observe the A/B-states with our probe wavelength in the TRPES, the pronounced vibrational structure observed in the absorption spectra ${ }^{143-147}$ indicates that these states are long-lived, in agreement with the non-adiabatic dynamics calculations and the experimentally observed fluorescence. Our work on the benzyl radical shows beautifully how different probe wavelengths illuminate different timescales, but they also illustrate how involved and non-"black-box" the description of its excited states are: in the benzyl case TDDFT descriptions of the excited states consistently failed to reproduce the energetic ordering of the Rydberg states relative to the $\pi \pi^{*}$-states (cf. Supplementary Material to Ref. ${ }^{120}$ ), showing that the electronic method has to be chosen with care. Thus, even though such calculations are computationally feasible, comparison to experimental data remains indispensable. 


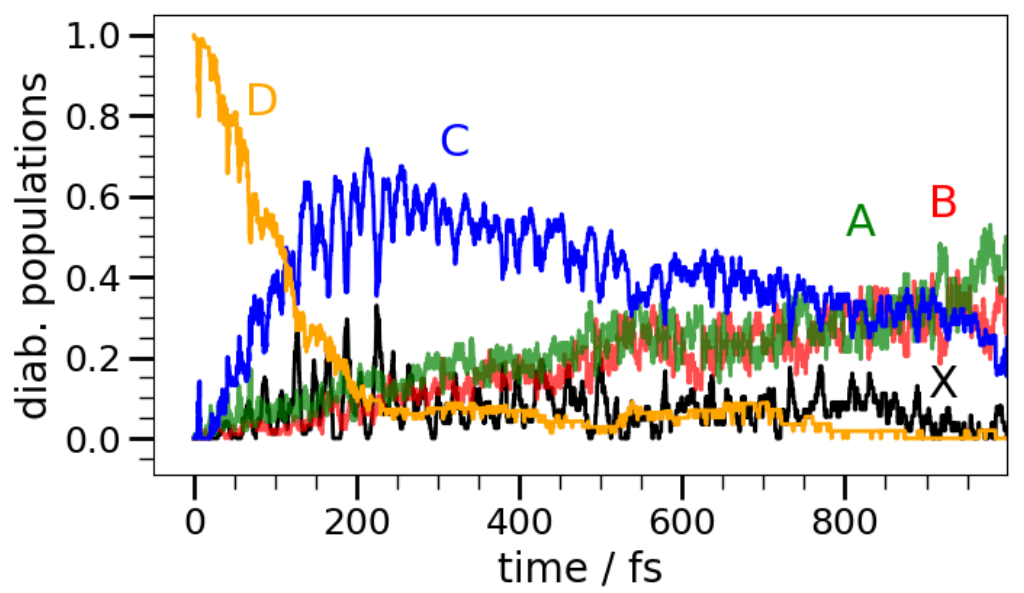

Figure 7. Diabatic populations following vertical excitation to $\mathrm{D}\left(\pi-\pi^{*}\right)$ of the benzyl radical, adapted with permission from Ref. ${ }^{120}$. Copyright 2017 Physical Chemistry Chemical Physics.

The TRPES of the tropyl radical (Figure 6, right) looks distinctively different from the one of the benzyl radical. Tropyl was produced via pyrolysis from bitropyl, synthesized according to literature $^{162}$. For photoexcitation, a wavelength of $266 \mathrm{~nm}$ was employed, while for the probe step either 800 or $400 \mathrm{~nm}$ were used. The IE of the tropyl radical is low $\left(6.23 \mathrm{eV}^{129,141,155,163,164}\right)$, two $800 \mathrm{~nm}$ probe photons or one $400 \mathrm{~nm}$ probe photon are already sufficient for ionization. No photoelectrons originating from higher order processes were observed in either the $800 \mathrm{~nm}$ or $400 \mathrm{~nm}$ spectra. Dissociative photoionization was visible through ion imaging and is explained in detail in the Supplementary information. For the $800 \mathrm{~nm}$ probe (Figure 6, right, upper panel) the decay can be fitted to a mono-exponential decay with $\tau_{1}=432 \mathrm{fs}$. With $400 \mathrm{~nm}$ probe (cf. SI, Fig. S5) the signal to noise ratio is considerably lower, and we can extract a mono-exponential decay with $\tau_{1}=$ $190 \mathrm{fs}$. The photoelectron spectrum with $400 \mathrm{~nm}$ probe is considerably less structured than the photoelectron spectrum with $800 \mathrm{~nm}$, showing only one broad band between 1.5 and $0 \mathrm{eV}$, indicating that the additional peaks visible in the $800 \mathrm{~nm}$ spectrum again are due to intermediate resonances in the probe step. This also explains the differences in the observed decay times. 
According to the results of our ab initio calculations summarized in the SI, Table S9, the optically bright excited states situated the closest to the experimental excitation energy of $4.65 \mathrm{eV}$ are of $\pi \pi^{*}$ and Rydberg $3 \mathrm{~d}$ character. This is consistent with the photoelectron anisotropy $\beta_{2}$ between 0.5 and 1 observed in the one-photon probe experiment.

Based on the available experimental data a firm conclusion on the relaxation dynamics of tropyl is difficult. Nevertheless, it seems that the initial state excited at $266 \mathrm{~nm}$ rapidly deactivates due to coupling with the manifold of close-lying lower states and leaves the observational window within roughly 400 fs.

Both for tropyl and benzyl, the excited state relaxation is strongly influenced by the coupling of valence and low-lying Rydberg states. The latter, which can be modeled by a single electron turning around an ionic core, have been proposed as efficient mediators between excited states ${ }^{165}$, since they cover the whole molecule and therefore overlap with all the valence states. This observation also applies to the case of benzyl and tropyl, but for the latter it is especially pronounced since many of the lower excited-states are indeed Rydberg states, which contribute to the faster relaxation of tropyl compared to benzyl. However, to fully understand the excited-state dynamics of the tropyl radical, further experiments (with different probe wavelengths) and highly accurate $a b$ initio dynamics simulations are necessary.

\subsubsection{Comparison of the benzyl relaxation dynamics to the xylyl radicals}

Formal replacement of a hydrogen atom in the phenyl ring of benzyl by a methyl group gives rise to the xylyl radicals, which exist as ortho-, meta-, and para isomers depending on the methyl position relative to the benzyl $\mathrm{CH}_{2}$ group. Recently Steglich et al. examined the ultrafast deactivation of the $\mathrm{D}_{3}\left({ }^{2} \mathrm{~A} ", 4 \mathrm{eV}\right)$-state of these three species using TRPES ${ }^{121}$. The xylyl radicals were 
produced via flash pyrolysis from bromide precursors. Similar to the benzyl radical, a bisequential decay was observed after excitation with $310 \mathrm{~nm}$. The deactivation rates of the ortho $\left(\tau_{1}=200 \pm\right.$ $\left.50 \mathrm{fs}, \tau_{2}=5.7 \pm 0.8 \mathrm{ps}\right)$ and para $\left(\tau_{1}=350 \pm 50 \mathrm{fs}, \tau_{2}=5.4 \pm 0.7 \mathrm{ps}\right)$-isomers are comparable with those of the benzyl radical excited to the $\mathrm{C}$ state origin at $305 \mathrm{~nm}\left(\tau_{1}=400, \tau_{2}=4.5 \mathrm{ps}\right)^{152}$, whereas the meta-xylyl deactivates significantly faster $\left(\tau_{1}<100, \tau_{2}=2.1 \pm 0.1 \mathrm{ps}\right)$. From a pure symmetry argument, the substitution of a methyl group in para-position should have the least influence on the dynamics, and its dynamics is the closest to the benzyl. For meta- and ortho-xylyl, however, one would expect slower decays due to larger energy pseudorotational barriers and lower state densities, as the methyl group rotates upon $\mathrm{D}_{0} / \mathrm{D}_{3} \operatorname{transition}^{121}$. In the benzyl radical, the conical intersections were caused by planar ring deformations ${ }^{120}$, which could be stabilized differently depending on the substitution pattern. Accurate high-level computations of the conical intersections are needed to provide more insight as to why the meta-isomer deactivates faster than the other two isomers. The photoelectron spectra were also dominated by intermediate Rydberg resonances due to the multi-photon probe (up to three $1.6 \mathrm{eV}$ photons), and no reasonable level of contrast could be achieved with a one-photon probe. However, this illustrates again that the relative

position of the methyl group considerably influences the dynamics via the 'inertial effect' ${ }^{11}$, meaning that there are specific vibrational motions which speed up the passage through the conical intersection(s).

\subsection{The resonantly stabilized biradicaloid para-xylylene}

Abstracting one more hydrogen from the xylyl radicals leads to the class of the xylylene molecules. Formally, these can be described either as biradicals, with an aromatic 6-ring augmented 
by two methylene radical centers, or as closed-shell quinoidal systems. The actual amount of biradicality present in these molecules strongly depends on the relative position of the two methylene groups. While the meta-xylylene has been identified to possess strong biradical character, ortho- and para-xylylene are better described as closed shell, quinoidal systems ${ }^{166}$. For para-xylylene, which will be focused on in the present section, the quinone ground state has been consolidated by electron diffraction ${ }^{167}, \mathrm{UV} / \mathrm{IR}^{168}$ and several theoretical studies ${ }^{169-171}$. Compared to the structurally related benzyl radical, its IE is higher $\left(7.87 \pm 0.05 \mathrm{eV}^{172,173}\right)$, though still lower than for the aromatic closed-shell para-xylene $\left(8.445 \pm 0.015 \mathrm{eV}^{174}\right)$. Just as for the benzyl radical, we performed a joint theoretical and experimental investigation of the excited-state dynamics of paraxylylene ${ }^{175}$.

At first glance, the excited states of para-xylylene in the experimentally relevant energy range up to $\sim 5 \mathrm{eV}$ are similar to those of the benzyl radical, as there are states of $\pi \pi^{*}$ - and $\mathrm{s} / \mathrm{p}$-Rydberg character present. However, as a remarkable difference, while in benzyl the brightest absorption can be attributed to close-lying Rydberg $3 p$ and $\pi \pi^{*}$ states, with additional Rydberg and $\pi \pi^{*}$ states situated below, in para-xylylene the lowest bright excited state has $\pi \pi^{*}$-character and lies below the lowest Rydberg manifold as shown by high-level ab initio calculations ${ }^{175}$. Therefore, in contrast to benzyl, Rydberg states will not strongly influence the dynamics of para-xylylene after excitation with $266 \mathrm{~nm}$. To unveil the excitation and subsequent relaxation dynamics we performed FISH simulations in the framework of the semiempirical multireference configuration interaction method ${ }^{176,177}$ employing the OM3 parameterization ${ }^{178,179}$, which very well reproduces the experimental $\pi \pi^{*}$ excitation energies ${ }^{175}$. Excitation of para-xylylene using $266 \mathrm{~nm}$ light mainly populates a bright $\pi \pi^{*}$ state, which is the second or third excited state $\left(\mathrm{S}_{2} / \mathrm{S}_{3}\right)$ depending on the actual molecular geometry. Subsequently, ultrafast relaxation to the $S_{1}$ state, which has 
doubly excited $\pi \pi^{*}$ character, occurs on a time scale of $40 \mathrm{fs}$, followed by the return to the ground state in several hundred femtoseconds ${ }^{175}$. This sequential relaxation of $\mathrm{S}_{2} / \mathrm{S}_{3}$ via $\mathrm{S}_{1}$ to the ground state could be nicely observed experimentally in the TRPES as well as in the mass spectra ${ }^{175}$. In the latter, the time-delayed appearance of ionic fragments (loss of hydrogen or a fragment with $\mathrm{m} / \mathrm{z}=26$ ) is observed, which is attributed to dissociative ionization specific for ionization of the $\mathrm{S}_{1}$ state. As illustrated in Figure 8 , the time-evolution of the experimental total photoion yield compares very well with the computed photoion intensities, which have been obtained by calculating the energy of the para-xylylene cation along the nuclear trajectories and assuming ionization to take place in all cases where the probe photon energy surmounted the current IE.

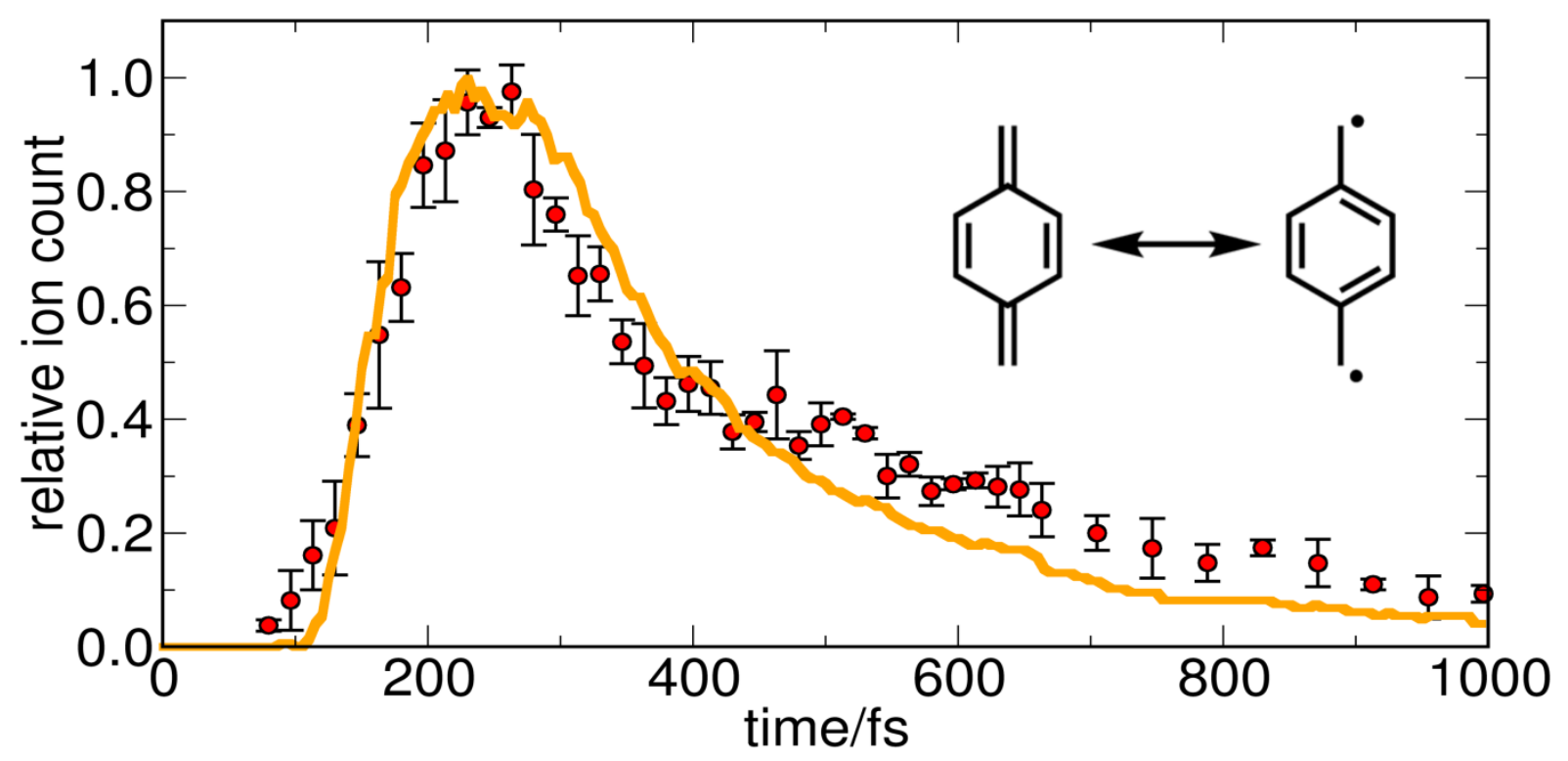

Figure 8. Comparison of experimental ion intensities of para-xylylene for $266 \mathrm{~nm}$ excitation and $794 \mathrm{~nm}$ ionization (red dots and error bars) with the simulated time-resolved ion intensities (orange) based on the FISH simulation. Reproduced with permission from Ref ${ }^{175}$. Copyright 2018 Faraday Discussions. 
It is interesting to note that both electronic excitation and vibrational motion have a strong influence on the biradical character of para-xylylene, as has been quantified by computing the natural orbital occupation numbers $(\mathrm{NOONs})^{166,180}$ along the trajectories ${ }^{175}$. For a perfect biradical, these numbers should be unity for exactly two natural orbitals, while for the others, values of zero or two are expected. In its electronic ground state, para-xylylene only has low biradical character, with the NOONs deviating the most from 0 or 2 assuming average values of 0.2 or 1.8 , respectively. After excitation with $266 \mathrm{~nm}$ into the $\mathrm{S}_{2} / \mathrm{S}_{3} \pi \pi^{*}$-state the biradical character significantly increases, with values of the aforementioned NOONs of 0.7 or 1.3 on average, reflecting the singly-excited character of the bright $\pi \pi^{*}$ state. However, even after return to the ground state an increased biradical character persists on the time scale of $1 \mathrm{ps}$. This is due to stronger vibrational motion caused by increased nuclear kinetic energy, leading the molecule more frequently to distorted, more biradical geometries.

This combined experimental and theoretical work illustrates that we can establish the link between characteristics of the electronic structure and the dynamical processes, allowing us to follow the temporal evolution of the open-shell character after photoexcitation. We anticipate that the observed increase in biradicality after excitation has considerable influence on the chemical reactivity, which might be exploited in organic synthesis and deserves further investigation.

\subsection{The reactive carbene cyclopropenylidene}

Besides radicals and biradicals, in which one or two unpaired electrons are responsible for increased reactivity, another reactive class of organic molecules is represented by singlet carbenes. In these systems, the electronic structure can be characterized by a nonbonding electron pair and an empty p-orbital localized at a carbon atom. This particular situation makes singlet carbenes 
prone both to reactions with electrophilic and nucleophilic reaction partners. In nature, carbenes play an important role in the chemistry of interstellar space ${ }^{181}$, where cyclopropenylidene (c$\mathrm{C}_{3} \mathrm{H}_{2}$,) is one of the most abundant hydrocarbons ${ }^{181,182}$. Especially in the interstellar medium, where radiation is omnipresent, an understanding of the photophysics and deactivation processes of its excited states is necessary to develop further models for the formation of complex molecules in outer space. Previously both the IE and ionization cross sections have been determined ${ }^{183}$ and the electronic spectrum of the $\mathrm{B}^{1} \mathrm{~B}_{1}$-state was measured ${ }^{184}$, before the excited-state dynamics of this reactive carbene using TR-PES ${ }^{116}$ has been explored. While in the B-state an electron is promoted from the carbene lone pair to the empty p-orbital located at the carbene center, in the lowerlying A-state the electron is excited into the $\pi^{*}$ orbital of the C-C double bond ${ }^{184}$.

Cyclopropenylidene was produced by pyrolysis of 3-chlorocycloprop-1-ene and excited at 265 $\mathrm{nm}$ into the $\mathrm{B}$-state ${ }^{184}$. The dynamics was then probed by $800 \mathrm{~nm}$ multiphoton ionization. As the IE is $9.17 \mathrm{eV}$, a minimum of three photons is necessary for ionization. However, [1+4'] processes dominate in the TRPES depicted in Figure 9. 


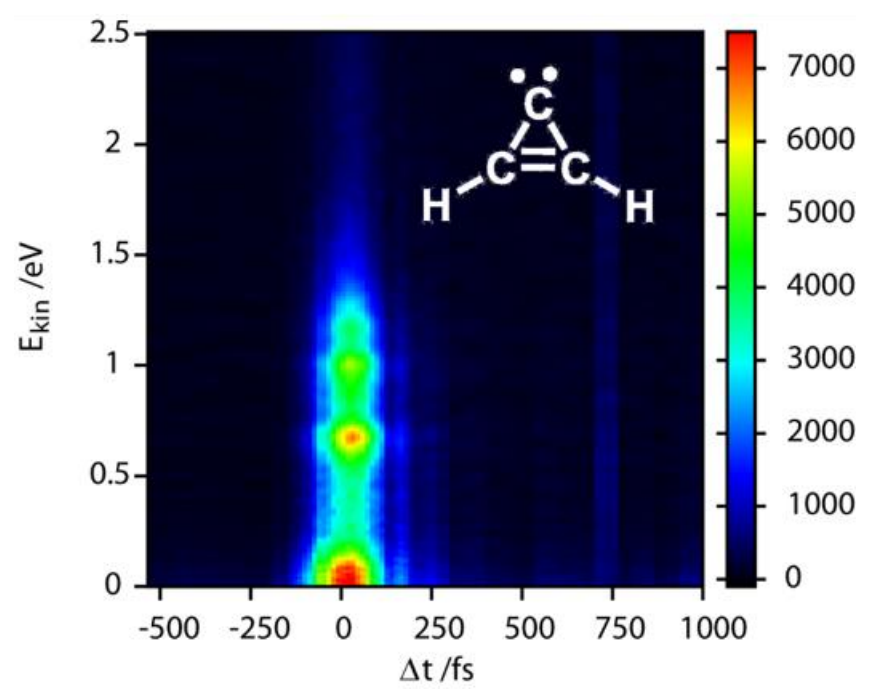

Figure 9. TRPES of cyclopropenylidene excited with $265 \mathrm{~nm}$ and probed with $800 \mathrm{~nm}$ multiphoton ionization. Adapted from Ref ${ }^{116}$. Copyright Physical Chemistry Chemical Physics.

The B state, previously observed using REMPI spectroscopy ${ }^{184}$, was also identifiable in the photoelectron spectra via its characteristic Rydberg resonances. Since the electron is ejected from an almost pure p-orbital, ionization proceeds through intermediate d-Rydberg resonances, as could be inferred from the peak positions in the TRPES (cf. Figure 9 and ${ }^{116}$ ). An ultrafast decay of the B state was observed, showing two time-constants of $\tau_{1}<50 \mathrm{fs}$ and $\tau_{2}=180 \mathrm{fs}^{116}$. The short time-constant was attributed to the rapid initial decay of part of the wavepacket via the A-state to the ground state. The second time-constant, however, reflects components of the wavepacket which are deflected by the initial passage through the conical intersection ${ }^{185}$ and remain in the Bstate for a longer time. Once in the ground state, loss of a hydrogen atom occurs, forming both c$\mathrm{C}_{3} \mathrm{H}$ and its linear isomer $1-\mathrm{C}_{3} \mathrm{H}$, as has been observed in nanosecond-photodissociation experiments $^{185}$.

\section{Alkyl Radicals: tert-Butyl}


The photophysics and photochemistry of alkyl radicals is still not well understood, although a considerable amount of experimental work is available, focusing mostly on the photodissociation products ${ }^{186,187}$. However, so far only the tert-butyl radical $\mathrm{C}\left(\mathrm{CH}_{3}\right)_{3}$ has been examined using TRPES.

This system is structurally somewhat similar to the 2-MA radical $\mathrm{C}\left(\mathrm{CH}_{2}\right)_{2} \mathrm{CH}_{3}$ discussed before, and can be considered its "saturated analog", formally generated by adding one hydrogen atom at each methylene group. In doing so, from the former $\pi$-system only a single unpaired electron remains, which is localized in a $2 p$-orbital at the central carbon. Since no $\pi$-orbitals are present anymore and the virtual $\sigma$-orbitals lie quite high in energy, the low-lying excited states of tertbutyl are all of Rydberg character ${ }^{19}$.

The first excited state at $\sim 3.7 \mathrm{eV}$ is thus a $3 \mathrm{~s}$ Rydberg state, and the next two higher-lying states have $3 p$ and $3 d$ Rydberg character, which have been observed and assigned employing absorption spectroscopy ${ }^{19}$ and ab-initio quantum chemistry ${ }^{188}$. All valence excited states have $\sigma^{*}$ character and are very high in energy at the ground state equilibrium geometry. Upon excitation the $\mathrm{C}_{3 \mathrm{v}^{-}}$ symmetric tert-butyl radical changes its geometry from pyramidal to a planar carbon framework and approaches the ground state structure of the ion.

In the experiment, tert-butyl was cleanly and efficiently generated from azobutane, and the A $3 \mathrm{~s}$ state was excited with $307-347 \mathrm{~nm}$ and ionized using $810 \mathrm{~nm}$ in a [1+2'] and [1+3']-process ${ }^{189}$. The photoelectron images given in Figure 10 are dominated by a polarized contribution of $p$ character and confirm that ionization occurs from an almost pure s-state. 


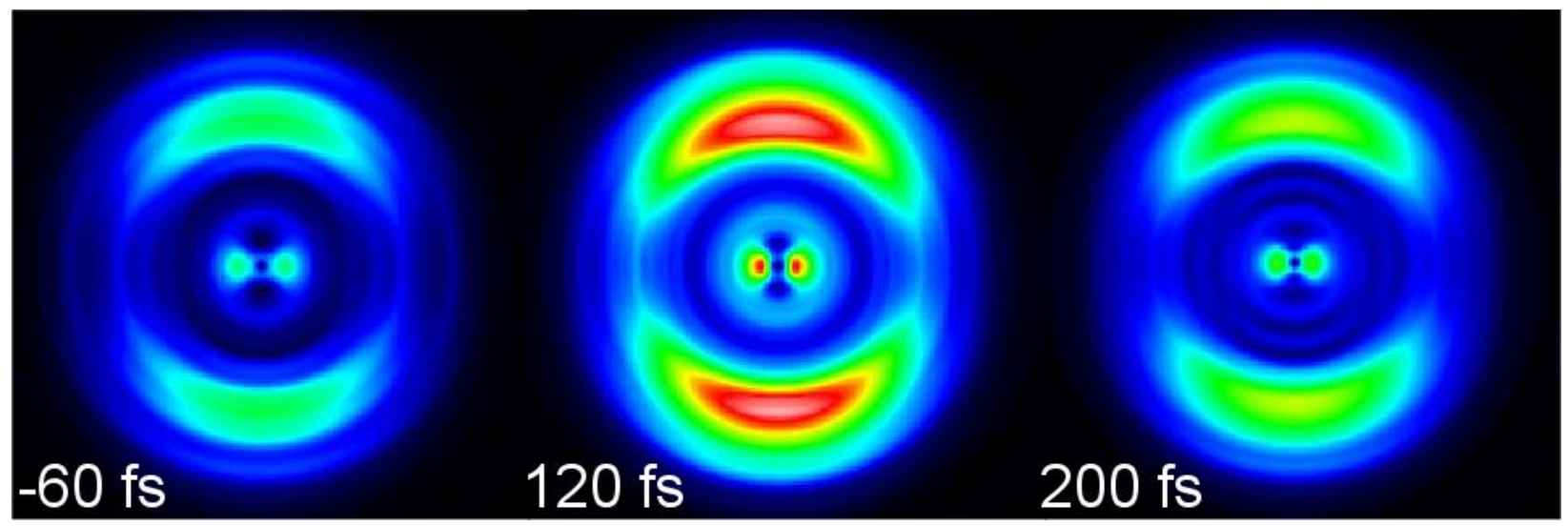

Figure 10. Photoelectron images at selected pump-probe delays, recorded at $324 \mathrm{~nm}$, corresponding to excitation into the $\mathrm{A}^{2} \mathrm{~A}_{1}(3 \mathrm{~s})$ state. Figure reproduced from ${ }^{189}$. Copyright Journal of Physical Chemistry A.

Interestingly, the photoelectron spectra remain virtually unchanged when tuning the excitation energy between $307-347 \mathrm{~nm}$. Due to the geometry change from pyramidal to planar, excitation to the $3 \mathrm{~s}$ state will deposit a considerable amount of energy in the umbrella mode. Since the geometries of the excited state and the ion are similar, this vibrational excitation will be transferred into the ion. However, given the relatively low kinetic energy of the observed photoelectrons ${ }^{189}$, the cation must have been formed with even larger vibrational energy than expected due to the pump excitation. This effect is probably caused by efficient enhancement of intermediate Rydberg resonances in the probe step as has been observed before ${ }^{118,190}$. The signature of the A $3 \mathrm{~s}$-state decays quickly, with a time constant of $180 \mathrm{fs}$ for the lowest excitation energy corresponding to $347 \mathrm{~nm}$. A drop of the lifetime down to $69 \mathrm{fs}$ is observed for excitation wavelengths below $330 \mathrm{~nm}^{189}$. The rapid decay of the $3 \mathrm{~s}$ state suggests a relaxation via a conical intersection to the electronic ground state. These hot ground-state tert-butyl radicals then lose an $\mathrm{H}$ atom on a nanosecond time-scale, as shown in earlier experiments ${ }^{191}$. In that study a drop in the dissociation rate for excitation wavelengths below $330 \mathrm{~nm}$ was observed, which coincides with the drop of lifetime discussed above. 
It is likely that at such higher excitation energies another decay channel becomes accessible, which may involve the higher-lying and longer-lived $3 p$ state (see below), and cleavage of a C-C bond after a [1,2]-H-shift, leading to the formation of propene as a possible dissociation pathway ${ }^{191}$.

With 268 or $274 \mathrm{~nm}$ the three $3 p$ Rydberg states (exhibiting $A_{1}$ and E symmetry, respectively) were excited, and in the probe step two $810 \mathrm{~nm}$ photons were used ${ }^{189}$. The $3 p$ states lie so close together that they cannot be separated in absorption spectroscopy ${ }^{192}$. In the photoelectron spectrum a peak at $0.44 \mathrm{eV}$ is observed, corresponding to the formation of ions with a considerable internal energy of $0.66 \mathrm{eV}$. The whole photoelectron spectrum decays uniformly following a mono-exponential decay with $\tau_{1}=2 \mathrm{ps}$. Considering the much faster decay from the $3 \mathrm{~s}$ state as well as the fact that the $3 p$ absorption band is very broad ${ }^{192}$, such a long decay is rather surprising. Presumably a very fast population flow within the manifold of the $3 p$ states occurs, which only after some time continues onwards to the $3 \mathrm{~s}$ or the ground state. This may hint at a local minimum on one of the $3 p$ state potential energy surfaces, but further theoretical investigations are necessary to draw a firm conclusion. For both excitation to $3 \mathrm{~s}$ and $3 p$ the experimental signal decays exponentially, and no additional band growing in time was observed.

Notably, after excitation to the $3 p$ band using higher energy (excitation wavelength of $248 \mathrm{~nm}$ ), the loss of a methyl group was observed by photofragment translational spectroscopy, and was attributed to a direct dissociation in the excited state leading to dimethylcarbene ${ }^{187}$. As a tentative explanation, the interaction of the $3 \mathrm{~s}$ and $3 p$ Rydberg states with a dissociative valence state was proposed ${ }^{187}$. Although such a $\sigma^{*}$ excited valence state is inaccessible from the ground state equilibrium, it will decrease in energy upon stretching a $\mathrm{C}-\mathrm{C}$ bond, because it corresponds to the dissociative coordinate to dimethylcarbene $+\mathrm{CH}_{3}$. This hypothesis has been further developed in an 
attempt to rationalize the results of several $\mathrm{H}$-atom photofragment imaging studies on the photodissociation dynamics of alkyl radicals ${ }^{186}$, but high-level theoretical studies taking Rydberg/valence interaction into account are needed for further insight.

The relaxation dynamics of the $3 p$ states of the tert-butyl radical can be compared to its more sterically hindered analogue, the 2,3-dimethylbut-2-yl (DMB) radical, in which one of the methyl groups is replaced by an isopropyl group. So far, this molecule has been investigated using timeresolved mass spectroscopy ${ }^{19}$, as it could not be produced sufficiently cleanly to measure TRPES so far. DMB shows a biexponential decay with $\tau_{1}<25 \mathrm{fs}$ and $\tau_{2}=400 \mathrm{fs}$, considerably faster than in the case of the tert-butyl radical. Unlike tert-buytl, DMB does not exhibit $\mathrm{C}_{3 \mathrm{v}}$ symmetry, thus transitions and deactivation pathways which are symmetry-forbidden in $\mathrm{C}_{3 \mathrm{v}}$ now become accessible, leading to faster relaxation times. This effect has been observed before in closed-shell molecules such as substituted benzene derivatives ${ }^{193}$. Also the higher vibrational state density of DMB due to the replacement of a methyl by an isopropyl group probably plays a role in the increase of the excited-state relaxation. Comparing the relaxation dynamics of tert-butyl with other radicals especially the methyl and iso-propyl radicals would provide fascinating insights into how lowering Rydberg states change the dynamics, however, such experiments have not been performed yet as clean production of these small hydrocarbons is an unsolved challenge.

\section{Discussion}

In general, for all reactive hydrocarbons discussed above we observed a fast deactivation after UV excitation followed by dissociation in the ground state. This is often followed by ejection of a hydrogen atom, as was seen in the cases of e.g. the allyl and the benzyl radical. No general ten- 
dency in deactivation speed according to size can be drawn, however, as e.g. both cyclopropenylidene and the allyl radical (both three carbon atoms) differ vastly, as do the benzyl and tropyl radical.

TRPES of open-shell molecules provide a fascinating insight on the excited-state dynamics of these short-lived intermediates. One of the big challenges with TRPES methods applied so far is the clean production in situ, and neither possible side products nor the precursor should show excited-state dynamics in the experimental wavelength region. That is one of the main reasons why so many TRPES (in contrast to time-resolved mass spectrometry) of hydrocarbon open-shell molecules, ranging from small systems like ethyl and propargyl to larger polycyclic aromatic hydrocarbons, remain unexplored, even though such molecules are of paramount importance in various fields such as combustion processes ${ }^{3}$, soot formation ${ }^{194}$, synthesis of carbon nanomaterials ${ }^{195}$, astrochemistry ${ }^{4,5,196}$. Time-resolved photoelectron photoion coincidence (TR-PEPICO) is one technique bypassing the difficulties of clean production: Here photoelectron and photoions are detected in coincidence, allowing the recording of mass-selective photoelectron spectra. Such experiments, however, are due to their long acquisition times challenging in their own right, but they have provided valuable insight into the excited-state dynamics of closed-shell molecules ${ }^{22-24}$.

To achieve a deep molecular-level understanding of the excited-state dynamics of open-shell species, collaboration of experiment and theory is mandatory. In particular, the examples presented above impressively show how the simulation of time-resolved spectra based on coupled electronnuclear dynamics allows one to extract valuable information about dynamical mechanisms, involved electronic states and possible reaction pathways, which can be connected to the experimentally observed data. At the same time, the validation of the employed theoretical approach is made possible by comparison between calculated and measured observables. Indeed, the electronic 
structure method to calculate the energetics and character of the participating electronically excited states needs to be chosen with care, as, e.g., in the case of the benzyl radical, only by thorough analysis of several electronic structure methods we were able to elucidate if and how Rydberg states are involved in the dynamics. The right energetic positions of Rydberg states can be particularly difficult to describe with a computationally efficient method such as TDDFT, though this depends heavily on the particular open-shell species. In addition, the presence of states with double excitation character, such as the $\mathrm{S}_{1}$ state of para-xylylene, prevents the use of TDDFT as well.

Future improvement of the available experimental methodology will include using freely tunable ultrashort pump lasers and higher-energetic ultrashort probe lasers, thus providing additional insight into the excited-state dynamics: while the former allow one to more selectively choose the quantum state in which the dynamics is initiated, including mode-specific vibrational excitation, the latter widen the observational window of the dynamics. Recently $14 \mathrm{eV}^{56,197}$ and $7.7 \mathrm{eV}^{198}$ probe pulses have been used to record TRPES of closed-shell molecules. Due to the low ionization potential of open-shell hydrocarbon molecules, using such wavelengths to record their TRPES would allow measuring the complete excited-state dynamics and even observing the ground state in a one-photon ionization step. The interpretation of such [1+1'] ionization processes is not only much simpler than those of multi-photon ionization processes, but the corresponding PADs can be calculated using theoretical methods, such as presented in the Methods section ${ }^{52}$, thus providing additional insights.

It is our hope that by continuing to explore the excited-state dynamics of open-shell hydrocarbon molecules, especially with systematic structural variations, a general set of rules for the underlying relaxation mechanisms will be elaborated, effectively linking geometric and electronic structure as well as interstate coupling and dynamical relaxation time scales. 
In conclusion, we reviewed our recent work on the TRPES of reactive hydrocarbon molecules, showing that while both experiments and simulations on these systems are challenging, they provide valuable insight in the dynamics of these short-lived intermediates. We are convinced that through continuous improvement of experimental techniques the excited-state dynamics of other open-shell hydrocarbon species will be further elaborated in the future.

\section{Acknowledgments}

We wish to thank our coworkers involved in the various studies reviewed in this Feature Article, in particular Alexander Humeniuk, who developed the theoretical methodology for the simulation of time-resolved photoelectron imaging, and the laser team that operates the LUCA laser of the SLIC laser facility, especially Olivier Gobert for tunability on demand.

Large parts of this work were supported by the Deutsche Forschungsgemeinschaft, Research Training School, GRK 2112 "Molecular Biradicals: Structure, Properties and Reactivity”. AR, JP and KI thank the COST action CM1405 MOLIM for support. RM acknowledges the support within the ERC Consolidator Grant "DYNAMO" (Grant No. 646737). LP and IF acknowledge funding by DAAD and Campus France, PHC PROCOPE 32980XH and support from LaserlabEurope (EU-H2020 654148). Finally, LP acknowledges ANR11-EQPX0005-ATTOLAB for support.

\section{Supporting Information}

Details on the excited states of 1MA, the NTO analysis for 2MA, a comparison between the experimental and theoretical state assignments for allyl, 1MA and 2MA, an analysis of the kinetic energy of 1MA during FISH, details concerning the excited state calculations of the tropyl 
radical and the experimental TRPES of tropyl ionized with $400 \mathrm{~nm}$ and an analysis of dissociative photoionization of the tropyl radical can be found in the supplementary information.

\section{References}

(1) Zewail, A. H. Femtochemistry: Atomic-Scale Dynamics of the Chemical Bond Using Ultrafast Lasers (Nobel Lecture). Angew. Chemie - Int. Ed. 2000, 39, 2586-2631.

(2) Benson, S. W. Oxygen Initiated Combustion: Thermochemistry and Kinetics of Unsaturated Hydrocarbons. Fuel Energy Abstr. 1997, 38 (2), 108. https://doi.org/10.1016/S0140-6701(97)83146-3.

(3) Glassman, I.; Yetter, R. A. Combustion, 4th ed.; 2008.

(4) Tielens, A. G. G. M. The Physics and Chemistry of the Interstellar Medium; Cambridge University Press, 2005.

(5) Ehrenfreund, P.; Charnley, S. B. Organic Molecules in the Interstellar Medium, Comets, and Meteorites: A Voyage from Dark Clouds to the Early Earth. Annu. Rev. Astron. Astrophys. 2000, 38 (1), 427-483. https://doi.org/10.1146/annurev.astro.38.1.427.

(6) McGuire, A. D.; Anderson, L. G.; Christensen, T. R.; Dallimore, S.; Guo, L.; Hayes, D. J.; Heimann, M.; Lorenson, T. D.; Macdonald, R. W.; Roulet, N. Sensitivity of the Carbon Cycle in the Arctic to Climate Change. Ecol. Monogr. 2009, 79 (4), 523-555. https://doi.org/10.1890/08-2025.1.

(7) Krylov, A. I. The Quantum Chemistry of Open-Shell Species. In Reviews in Computational 
Chemistry; Parill, A. L., Lipkowitz, K. B., Eds.; 2017.

(8) Lochbrunner, S.; Larsen, J. J.; Shaffer, J. P.; Schmitt, M.; Schultz, T.; Underwood, J. G.;

Stolow, A. Methods and Applications of Femtosecond Time-Resolved Photoelectron Spectroscopy. J. Electron Spectros. Relat. Phenomena 2000, 112 (1-3), 183-198. https://doi.org/10.1016/S0368-2048(00)00212-7.

(9) Stolow, A.; Bragg, A. E.; Neumark, D. M. Femtosecond Time-Resolved Photoelectron Spectroscopy. Chem. Rev. 2004, 104 (4), 1719-1757. https://doi.org/10.1021/cr020683w.

(10) Suzuki, T. Femtosecond Time-Resolved Photoelectron Imaging. Annu. Rev. Phys. Chem. 2006, 57 (1), 555-592. https://doi.org/10.1146/annurev.physchem.57.032905.104601.

(11) Schuurman, M. S.; Stolow, A. Dynamics at Conical Intersections. Annu. Rev. Phys. Chem. 2018, 69, 427-450.

(12) Fischer, I.; Villeneuve, D. M.; Vrakking, M. J. J.; Stolow, A. Femtosecond Wave-Packet Dynamics Studied by Time-Resolved Zero-Kinetic Energy Photoelectron Spectroscopy. J. Chem. Phys. 1995, 102 (13), 5566. https://doi.org/10.1063/1.469285.

(13) Eiden, G. C.; Lu, K.; Badenhoop, J.; Weinhold, F.; Weisshaar, J. C. Threshold Photoionization Spectra of Benzyl Radical: Cation Vibrational States and Ab Initio Calculations. J. Chem. Phys. 1996, 104 (22), 8886-8895. https://doi.org/10.1063/1.471624.

(14) Lossing, F. P.; Ingold, K. U.; Henderson, I. H. S. Free Radicals by Mass Spectrometry. V. The Ionization Potentials of Methyl, Allyl, and Benzyl Radicals. J. Chem. Phys. 1954, 22 (4), 621-625. https://doi.org/10.1063/1.1740136. 
(15) Klasinc, L.; Kovac, B.; Güsten, H. Photoelectron Spectra of Acenes. Electronic Structure and Substituent Effects. Pure Appl. Chem. 1983, 55 (2), 289-298. https://doi.org/10.1351/pac198855020289.

(16) Luo, Y. R.; Pacey, P. D. Effects of Alkyl Substitution on Ionization Energies of Alkanes and Haloalkanes and on Heats of Formation of Their Molecular Cations Part 1. Simple Methyl Derivatives. Int. J. Mass Spectrom. Ion Process. 1992, 112, 63-77. https://doi.org/10.1016/0168-1176(91)85035-K.

(17) Tonokura, K.; Koshi, M. Cavity Ring-down Spectroscopy of the Benzyl Radical. J. Phys. Chem. A 2003, 107 (22), 4457-4461. https://doi.org/10.1021/jp027280k.

(18) Geppert, W. D.; Dessent, C. E. H.; Cockett, M. C. R.; Müller-Dethlefs, K. Evidence for Stable Rydberg States in the Presence of an Electron Scavenger: The ZEKE Spectrum of the Toluene-SF6 Complex. Chem. Phys. Lett. 1999, 303, 194-200. https://doi.org/10.1016/S0009-2614(99)00201-8.

(19) Noller, B.; Poisson, L.; Fischer, I.; Mestdagh, J. M. Side-Chain Effects on the Electronic Relaxation of Radicals Followed by Time-Resolved Pump-Probe Spectroscopy: 2,3Dimethylbut-2-Yl vs Tert-Butyl. J. Phys. Chem. A 2010, 114 (9), 3045-3049. https://doi.org/10.1021/jp9062059.

(20) Lombos, B. A.; Sauvageau, P.; C., S. The Electronic Spectra of Normal Paraffin Hydrocarbons. Chem. Phys. Lett. 1967, 42-43.

(21) Schmidt, T. W. The Electronic Spectroscopy of Resonance-Stabilised Hydrocarbon Radicals. Int. Rev. Phys. Chem. 2016, 35 (2), 209-242. 
https://doi.org/10.1080/0144235X.2016.1166830.

(22) Davies, J. A.; Leclaire, J. E.; Continetti, R. E.; Hayden, C. C. Femtosecond Time-Resolved Photoelectron - Photoion Coincidence Imaging Studies of Dissociation Dynamics. J. Chem. Phys. 1999, 111 (1), 1-5. https://doi.org/10.1063/1.479248.

(23) Davies, J. A.; Continetti, R. E.; Chandler, D. W.; Hayden, C. C. Femtosecond TimeResolved Photoelectron Angular Distributions Probed during Photodissociation of $\mathrm{NO}_{2}$. Phys. Rev. Lett. 2000, 84 (26), 5983-5986. https://doi.org/10.1103/PhysRevLett.84.5983.

(24) Hockett, P.; Ripani, E.; Rytwinski, A.; Stolow, A. Probing Ultrafast Dynamics with TimeResolved Multi-Dimensional Coincidence Imaging: Butadiene. J. Mod. Opt. 2013, 60 (17), 1409-1425. https://doi.org/10.1080/09500340.2013.801525.

(25) Willitsch, S.; Dyke, J. M.; Merkt, F. Generation and High-Resolution Photoelectron Spectroscopy of Small Organic Radicals in Cold Supersonic Expansions. Helv. Chim. Acta 2003, 86 (4), 1152-1166. https://doi.org/10.1002/hlca.200390100.

(26) Engelking, P. C. Corona Excited Supersonic Expansion. Rev. Sci. Instrum. 1986, 57 (9), 2274-2277. https://doi.org/10.1063/1.1138696.

(27) Kohn, D. W.; Clauberg, H.; Chen, P. Flash Pyrolysis Nozzle for Generation of Radicals in a Supersonic Jet Expansion. Rev. Sci. Instrum. 1992, 63 (8), 4003-4005. https://doi.org/10.1063/1.1143254.

(28) Blush, J. A.; Minsek, D. W.; Chen, P. Electronic Spectrum of Allyl and Allyl d 5 Radicals. The $\mathrm{B}\left[1^{2} \mathrm{~A}_{1}\right] \leftarrow \mathrm{X}\left[1^{2} \mathrm{~A}_{2}\right], \mathrm{C}\left[2^{2} \mathrm{~B}_{1}\right] \leftarrow \mathrm{X}\left[1^{2} \mathrm{~A}_{2}\right]$ and $\mathrm{B}\left[2^{2} \mathrm{~B}_{2}\right] \leftarrow \mathrm{X}\left[1^{2} \mathrm{~A}_{2}\right] . J$. Phys. Chem. 1992, 
96, 10150. https://doi.org/10.1021/j100204a013.

(29) Rijs, A. M.; Backus, E. H. G.; De Lange, C. A.; Westwood, N. P. C.; Janssen, M. H. M. 'Magnetic Bottle' Spectrometer as a Versatile Tool for Laser Photoelectron Spectroscopy. J. Electron Spectros. Relat. Phenomena 2000, 112 (1-3), 151-162. https://doi.org/10.1016/S0368-2048(00)00209-7.

(30) Parker, D. H.; Eppink, A. T. J. B. Photoelectron and Photofragment Velocity Map Imaging of State-Selected Molecular Oxygen Dissociation/Ionization Dynamics. J. Chem. Phys. 1997, 107 (7), 2357-2362. https://doi.org/10.1063/1.474624.

(31) Eppink, A. T. J. B.; Parker, D. H. Velocity Map Imaging of Ions and Electrons Using Electrostatic Lenses: Application in Photoelectron and Photofragment Ion Imaging of Molecular Oxygen. Rev. Sci. Instrum. 1997, 68 (9), 3477-3484. https://doi.org/10.1063/1.1148310.

(32) Imaging in Molecular Dynamics Technology and Applications; Whitaker, B., Ed.; Cambridge University Press, 2004.

(33) Hicks, P. J.; Daviel, S.; Wallbank, B.; Comer, J. An Electron Spectrometer Using a New Multidetector System Based on a Charge-Coupled Imaging Device. J. Phys. E. 1980, 13 (7), 713-715. https://doi.org/10.1088/0022-3735/13/7/004.

(34) Montgomery Smith, L.; Keefer, D. R.; Sudharsanan, S. I. Abel Inversion Using Transform Techniques. J. Quant. Spectrosc. Radiat. Transf. 1988, 39 (5), 367-373. https://doi.org/10.1016/0022-4073(88)90101-X. 
(35) Hansen, E. W.; Law, P.-L. Recursive Methods for Computing the Abel Transform and Its Inverse. J. Opt. Soc. Am. A 1985, 2 (4), 510. https://doi.org/10.1364/JOSAA.2.000510.

(36) Winterhalter, J.; Maier, D.; Honerkamp, J.; Schyja, V.; Helm, H. Imaging of Charged Atomic Reaction Products: Inversion by a Two-Dimensional Regularization Method. $J$. Chem. Phys. 1999, 110 (23), 11187-11196. https://doi.org/10.1063/1.479060.

(37) Dick, B. Inverting Ion Images without Abel Inversion: Maximum Entropy Reconstruction of Velocity Maps. Phys. Chem. Chem. Phys. 2014, 16 (2), 570-580. https://doi.org/10.1039/C3CP53673D.

(38) Garcia, G. A.; Nahon, L.; Powis, I. Two-Dimensional Charged Particle Image Inversion Using a Polar Basis Function Expansion. Rev. Sci. Instrum. 2004, 75 (11), 4989-4996. https://doi.org/10.1063/1.1807578.

(39) Cooper, J.; Zare, R. N. Angular Distributions of Photoelectrons. J. Chem. Phys. 1968, 58, 942-943. https://doi.org/10.1016/0009-2614(70)80104-X.

(40) Stolow, A.; Bragg, A. E.; Neumark, D. M. Femtosecond Time-Resolved Photoelectron Spectroscopy. 2004, 104, 1719-1757. https://doi.org/10.1021/CR020683W.

(41) Reid, K. L. Photoelectron Angular Distributions. Annu. Rev. Phys. Chem. 2003, 54 (1), 397424. https://doi.org/10.1146/annurev.physchem.54.011002.103814.

(42) Mitrić, R.; Petersen, J.; Bonačić-Koutecký, V. Laser-Field-Induced Surface-Hopping Method for the Simulation and Control of Ultrafast Photodynamics. Phys. Rev. A - At. Mol. Opt. Phys. 2009, 79 (5), 1-6. https://doi.org/10.1103/PhysRevA.79.053416. 
(43) Röhr, M. I. S.; Marciniak, H.; Hoche, J.; Schreck, M. H.; Ceymann, H.; Mitric, R.; Lambert, C. Exciton Dynamics from Strong to Weak Coupling Limit Illustrated on a Series of Squaraine Dimers. J. Phys. Chem. C 2018, 122 (15), 8082-8093. https://doi.org/10.1021/acs.jpcc.8b00847.

(44) Petersen, J.; Lindner, J. O.; Mitrić, R. Ultrafast Photodynamics of Glucose. J. Phys. Chem. B 2018, 122 (1), 19-27. https://doi.org/10.1021/acs.jpcb.7b08602.

(45) Röhr, M. I. S.; Petersen, J.; Wohlgemuth, M.; Bonačië-Koutecký, V.; Mitrï, R. Nonlinear Absorption Dynamics Using Field-Induced Surface Hopping: Zinc Porphyrin in Water. ChemPhysChem 2013, 14 (7), 1377-1386. https://doi.org/10.1002/cphc.201300053.

(46) Tomasello, G.; Wohlgemuth, M.; Petersen, J.; Mitrić, R. Photodynamics of Free and Solvated Tyrosine. J. Phys. Chem. B 2012, $116 \quad$ (30), 8762-8770. https://doi.org/10.1021/jp302179m.

(47) Petersen, J.; Mitrić, R. Electronic Coherence within the Semiclassical Field-Induced Surface Hopping Method: Strong Field Quantum Control in $\mathrm{K}_{2}$. Phys. Chem. Chem. Phys. 2012, 14 (23), 8299-8306. https://doi.org/10.1039/c2cp40747g.

(48) Petersen, J.; Wohlgemuth, M.; Sellner, B.; Bonaić-Koutecký, V.; Lischka, H.; Mitrić, R. Laser Pulse Trains for Controlling Excited State Dynamics of Adenine in Water. Phys. Chem. Chem. Phys. 2012, 14 (14), 4687-4694. https://doi.org/10.1039/c2cp24002e.

(49) Petersen, J.; Mitrić, R.; Bonačić-Koutecký, V.; Wolf, J. P.; Roslund, J.; Rabitz, H. How Shaped Light Discriminates Nearly Identical Biochromophores. Phys. Rev. Lett. 2010, 105 (7), 1-4. https://doi.org/10.1103/PhysRevLett.105.073003. 
(50) Hillery, M.; O’Connel, R. F.; Scully, M. O.; Wigner, E. P. Distribution Functions in Physics: Fundamentals. Phys Rep. 1984, 106, 121-167.

(51) Mitrić, R.; Petersen, J.; Wohlgemuth, M.; Werner, U.; Bonačić-Koutecký, V.; Wöste, L.; Jortner, J. Time-Resolved Femtosecond Photoelectron Spectroscopy by Field-Induced Surface Hopping. J. Phys. Chem. A 2011, 115 (16), 3755-3765. https://doi.org/10.1021/jp106355n.

(52) Humeniuk, A.; Wohlgemuth, M.; Suzuki, T.; Mitric, R. Time-Resolved Photoelectron Imaging Spectra from Non-Adiabatic Molecular Dynamics Simulations. J. Chem. Phys. 2013, 139 (13). https://doi.org/10.1063/1.4820238.

(53) Mitrić, R.; Werner, U.; Bonačić-Kouteck, V. Nonadiabatic Dynamics and Simulation of Time Resolved Photoelectron Spectra within Time-Dependent Density Functional Theory: Ultrafast Photoswitching in Benzylideneaniline. J. Chem. Phys. 2008, 129 (16). https://doi.org/10.1063/1.3000012.

(54) Yamamoto, Y. I.; Suzuki, Y. I.; Tomasello, G.; Horio, T.; Karashima, S.; Mitríc, R.; Suzuki, T. Time- and Angle-Resolved Photoemission Spectroscopy of Hydrated Electrons near a Liquid Water Surface. Phys. Rev. Lett. 2014, 112 (18), 1-5. https://doi.org/10.1103/PhysRevLett.112.187603.

(55) Tomasello, G.; Humeniuk, A.; Mitrić, R. Exploring Ultrafast Dynamics of Pyrazine by Time-Resolved Photoelectron Imaging. J. Phys. Chem. A 2014, 118 (37), 8437-8445. https://doi.org/10.1021/jp5017328.

(56) Adachi, S.; Schatteburg, T.; Humeniuk, A.; Mitrić, R.; Suzuki, T. Probing Ultrafast 
Dynamics during and after Passing through Conical Intersections. Phys. Chem. Chem. Phys. 2019. https://doi.org/10.1039/C8CP04426K.

(57) Gomberg, M. An Instance of Trivalent Carbon: Triphenylmethyl. J. Am. Chem. Soc. 1900, 22 (11), 757-771. https://doi.org/10.1021/ja02049a006.

(58) Mo, Y.; Lin, Z.; Wu, W.; Zhang, Q. Delocalization in Allyl Cation, Radical, and Anion. $J$. Phys. Chem. 1996, 100 (16), 6469-6474. https://doi.org/10.1021/jp9526612.

(59) Fischer, I.; Chen, P. Allyl-a Model System for the Chemical Dynamics of Radicals. J. Phys. Chem. A 2002, 106 (17), 4291-4300. https://doi.org/10.1021/jp013708o.

(60) Fessenden, R. W.; Schuler, R. H. Electron Spin Resonance Studies of Transient Alkyl Radicals. J. Chem. Phys. 1963, 39 (9), 2147-2195. https://doi.org/10.1063/1.1701415.

(61) Han, J.; Utkin, Y. G.; Chen, H.; Hunt, N. T.; Curl, R. F. High-Resolution Infrared Spectra of Jet-Cooled Allyl Radical $\left(\mathrm{CH}_{2}-\mathrm{CH}-\mathrm{CH}_{2}\right): v_{2}, v_{3}$, and $v_{14} \mathrm{C}-\mathrm{H}$ Stretch Vibrations. $J$. Chem. Phys. 2002, 116 (15), 6505. https://doi.org/10.1063/1.1459417.

(62) Getty, J. D.; Liu, X.; Kelly, P. B. Vibronic Coupling of the Allyl Radical Excited States. J. Phys. Chem. 1992, 96 (25), 10155-10160. https://doi.org/10.1021/j100204a014.

(63) Getty, J. D.; Kelly, P. B. Vibrational Analysis of the $\mathrm{X}^{2} \mathrm{~A}_{2}$ State of the Allyl Radical. Chem. Phys. 1992, 168 (2-3), 357-364. https://doi.org/10.1016/0301-0104(92)87169-A.

(64) Liu, X.; Getty, J. D.; Kelly, P. B. Resonance Raman Spectrum of the Allyl-d 5 Radical and the Force Field Analysis of the Allyl Radical. J. Chem. Phys. 1993, 99 (3), 1522. https://doi.org/10.1063/1.465320. 
(65) Getty, J. D.; Burmeister, M. J.; Westre, S. G.; Kelly, P. B. Examination of Allyl Radical Excited-State Dynamics and Ground-State Vibrational Frequencies by Ultraviolet Resonance Raman Spectroscopy. J. Am. Chem. Soc. 1991, 113 (3), 801-805. https://doi.org/10.1021/ja00003a011.

(66) Achkasova, E.; Araki, M.; Denisov, A.; Maier, J. P. Rotational Analysis of the $\tilde{A}^{2} B_{1} \leftarrow \tilde{X}^{2} A_{2}$ Electronic Spectrum of the Allyl Radical. Mol. Phys. 2005, 103 (11-12), 1555-1560. https://doi.org/10.1080/00268970500044830.

(67) DeSain, J. D.; Thompson, R. I.; Sharma, S. D.; Curl, R. F. The Rotationally Resolved Infrared Spectrum of the $v_{1}$ Stretch of the Allyl Radical. J. Chem. Phys. 1998, 109 (18), 7803. https://doi.org/10.1063/1.477426.

(68) Sappey, A. D.; Weisshaar, J. C. Vibronic Spectrum of Cold, Gas-Phase Allyl Radicals by Multiphoton Ionization. J. Phys. Chem. 1987, 91 (14), 3731-3736. https://doi.org/10.1021/j100298a004.

(69) Vajda, E.; Tremmel, J.; Rozsondai, B.; Hargittai, I.; Maltsev, A. K.; Kagramanov, N. D.; Nefedov, O. M. Molecular Structure of Allyl Radical from Electron Diffraction. J. Am. Chem. Soc. 1986, 108 (15), 4352-4353. https://doi.org/10.1021/ja00275a020.

(70) Wu, J. C.; Li, R.; Chang, J. L.; Chen, Y. T. Rydberg States of the Allyl Radical Observed by Two-Photon Resonant Ionization Spectroscopy. J. Chem. Phys. 2000, 113 (17), 72867291. https://doi.org/10.1063/1.1313560.

(71) Liang, C.-W.; Chen, C.-C.; Wei, C.-Y.; Chen, Y.-T. Two-Photon Resonant Ionization Spectroscopy of the Allyl-H5 and Allyl-d $\mathrm{d}_{5}$ Radicals: Rydberg States and Ionization 
Energies. J. Chem. Phys. 2002, 116 (10), 4162. https://doi.org/10.1063/1.1450553.

(72) Hudgens, J. W.; Dulcey, C. S. Observation of the $3 \mathrm{~s}^{2} \mathrm{~A}_{1}$ Rydberg States of Allyl and 2Methylallyl Radicals with Multiphoton Ionization Spectroscopy. J. Phys. Chem. 1985, 89 (15), 1505. https://doi.org/10.1021/j100254a039.

(73) Gasser, M.; Frey, J. A.; Hostettler, J. M.; Bach, A.; Chen, P. Vibronic Structure of the 3s and 3p Rydberg States of the Allyl Radical. J. Phys. Chem. A 2010, 114 (1), 4704-4711. https://doi.org/10.1016/j.jms.2010.07.008.

(74) Houle, F. A.; Beauchamp, J. L. Detection and Investigation of Allyl and Benzyl Radicals by Photoelectron Spectroscopy. J. Am. Chem. Soc. 1978, 100 (11), 3290-3294. https://doi.org/10.1021/ja00479a005.

(75) Fischer, I.; Schüßler, T.; Deyerl, H. J.; Elhanine, M.; Alcaraz, C. Photoionization and Dissociative Photoionization of the Allyl Radical, $\mathrm{C}_{3} \mathrm{H}_{5}$. Int. J. Mass Spectrom. 2007, 261 (2-3), 227-233. https://doi.org/10.1016/j.ijms.2006.09.023.

(76) Gasser, M.; Schulenburg, A. M.; Dietiker, P. M.; Bach, A.; Merkt, F.; Chen, P. SinglePhoton and Resonance-Enhanced Multiphoton Threshold Ionization of the Allyl Radical. $J$. Chem. Phys. 2009, 131 (1). https://doi.org/10.1063/1.3157185.

(77) Glaser, R.; Choy, G. S. C. Electron and Spin Density Analysis of Spin-Projected Unrestricted Hartree-Fock Density Matrixes of Radicals. J. Phys. Chem. 1993, 97 (13), 3188-3198. https://doi.org/10.1021/j100115a022.

(78) Paldus, J.; Veillard, A. Doublet Stability of Ab Initio Scf Solutions for the Allyl Radical. 
Mol. Phys. 1978, 35 (2), 445-459. https://doi.org/10.1080/00268977800100331.

(79) Takada, T.; Dupuis, M. Theoretical Study of the Allyl Radical: Structure and Vibrational Analysis. J. Am. Chem. Soc. 1983, 105 (7), 1713-1716.

(80) Cometta-Morini, C.; Ha, T.-K.; Oth, J. F. M. Ab Initio Study of the Vibrational Spectra of the Allyl and the Cyclopropyl Radicals. J. Mol. Struct. 1989, 188 (1-2), 79-94. https://doi.org/10.1016/0166-1280(89)85027-4.

(81) Szalay, P. G.; Csaszar, A. G.; Fogarasi, G.; Karpfen, A.; Lischka, H. An Ab Initio Study of the Structure and Vibrational Spectra of Allyl and 1, 4-Pentadienyl Radicals. J. Chem. Phys. 1990, 93, 1246.

(82) Mach, T. J.; King, R. A.; Crawford, T. D. A Coupled Cluster Benchmark Study of the Electronic Spectrum of the Allyl Radical. J. Phys. Chem. A 2010, 114 (33), 8852-8857. https://doi.org/10.1021/jp102292x.

(83) Luo, Y.; Song, L.; Wu, W.; Danovich, D.; Shaik, S. The Ground and Excited States of Polyenyl Radicals $\mathrm{C}_{2 \mathrm{n}-1} \mathrm{H}_{2 \mathrm{n}+1}(\mathrm{~N}=2-13)$ : A Valence Bond Study. ChemPhysChem 2004, 5 (4), 515-528. https://doi.org/10.1002/cphc.200300935.

(84) Aquilante, F.; Jensen, K. P.; Roos, B. O. The Allyl Radical Revisited: A Theoretical Study of the Electronic Spectrum. Chem. Phys. Lett. 2003, 380 (5-6), 689-698. https://doi.org/10.1016/j.cplett.2003.09.079.

(85) Yamaguchi, M. A CASSCF Study of Photochemical Cyclization of the First Excited $\tilde{A}^{2} B_{1}$ State of the Allyl Radical. J. Mol. Struct. THEOCHEM 1996, 365 (2-3), 143-149. 
(86) Castiglioni, L.; Bach, A.; Chen, P. Spectroscopy and Dynamics of A $\left[{ }^{2} B_{1}\right]$ Allyl Radical. Phys. Chem. Chem. Phys. 2006, 8 (22), 2591-2598. https://doi.org/10.1039/b602412b.

(87) Hostettler, J. M.; Castiglioni, L.; Bach, A.; Chen, P. Photochemical Deactivation Pathways of the A-State Allyl Radical. Phys. Chem. Chem. Phys. 2009, 11 (37), 8262-8265. https://doi.org/10.1039/b909830e.

(88) Ha, T.-K.; Baumann, H.; Oth, J. F. M. Ab Initio CI Study of the Electronic Spectrum of the Allyl Radical $\quad\left(\mathrm{CH}_{2}-\mathrm{CH}-\mathrm{CH}_{2}\right) . \quad J . \quad$ Chem. Phys. 1986, 85, 1538. https://doi.org/10.1063/1.446543.

(89) Deyerl, H. J.; Gilbert, T.; Fischer, I.; Chen, P. Kinetics and Dynamics in the Photodissociation of the Allyl Radical. J. Chem. Phys. 1997, 107 (8), 3329-3332. https://doi.org/10.1063/1.474704.

(90) Deyerl, H.-J.; Fischer, I.; Chen, P. Photodissociation Dynamics of the Allyl Radical. $J$. Chem. Phys. 1999, 110, 1450-1462. https://doi.org/10.1063/1.478020.

(91) Stranges, D.; Stemmler, M.; Yang, X.; Chesko, J. D.; Suits, A. G.; Lee, Y. T. UV Photodissociation Dynamics of Allyl Radical by Photofragment Translational Spectroscopy. J. Chem. Phys. 1998, $109 \quad$ (13), 5372-5382. https://doi.org/10.1063/1.477156.

(92) Gasser, M.; Frey, J. A.; Hostettler, J. M.; Bach, A. Vibronic Structure of the 3s Rydberg State of the 2-Methylallyl Radical. J. Phys. Chem. A 2010, 114 (1), 4704-4711. https://doi.org/10.1016/j.jms.2010.07.008. 
(93) Röder, A.; Issler, K.; Poisson, L.; Humeniuk, A.; Wohlgemuth, M.; Comte, M.; Lepetit, F.; Fischer, I.; Mitric, R.; Petersen, J. Femtosecond Dynamics of the 2-Methylallyl Radical: A Computational and Experimental Study. J. Chem. Phys. 2017, 147 (1), 013902. https://doi.org/10.1063/1.4974150.

(94) Lang, M.; Holzmeier, F.; Hemberger, P.; Fischer, I. Threshold Photoelectron Spectra of Combustion Relevant $\mathrm{C}_{4} \mathrm{H}_{5}$ and $\mathrm{C}_{4} \mathrm{H}_{7}$ Isomers. J. Phys. Chem. a 2015, 119 (17), 3995-4000. https://doi.org/10.1021/acs.jpca.5b02153.

(95) Gasser, M.; Frey, J. A.; Hostettler, J. M.; Bach, A. Probing for Non-Statistical Effects in Dissociation of the 1-Methylallyl Radical. Chem. Commun. 2011, 47 (1), 301-303. https://doi.org/10.1039/C0CC01899F.

(96) Chen, C. C.; Wu, H. C.; Tseng, C. M.; Yang, Y. H.; Chen, Y. T. One- and Two-Photon Excitation Vibronic Spectra of 2-Methylallyl Radical at 4.6-5.6 EV. J. Chem. Phys. 2003, 119 (1), 241-250. https://doi.org/10.1063/1.1576381.

(97) Callear, A. B.; Lee, H. K. Electronic Spectra of the Free Allyl Radical and Some of Its Simple Derivatives. Trans. Faraday Soc. 1968, 308. https://doi.org/10.1039/tf9686400308.

(98) Nakashima, N.; Yoshihara, K. Oscillator Strengths of the Ultraviolet Bands of Hot and Relaxed Methallyl , Allyl, and Methyl Radicals. Laser Chem. 1987, No. C, 177-196.

(99) Yanai, T.; Tew, D. P.; Handy, N. C. A New Hybrid Exchange-Correlation Functional Using the Coulomb-Attenuating Method (CAM-B3LYP). Chem. Phys. Lett. 2004, 393 (1-3), 5157. https://doi.org/10.1016/j.cplett.2004.06.011. 
(100) Hehre, W. J.; Ditchfield, K.; Pople, J. A. Self-Consistent Molecular Orbital Methods. XII. Further Extensions of Gaussian-Type Basis Sets for Use in Molecular Orbital Studies of Organic Molecules. J. Chem. Phys. 1972, 56 (5), 2257-2261. https://doi.org/10.1063/1.1677527.

(101) Clark, T.; Chandrasekhar, J.; Spitznagel, G. W. Efficient Diffuse Function- Augmented Basis Sets for Anion Calculations. III. The 3-21+G Basis Set for First-Row Elements, Li-F. J. Comput. Chem. 1983, 4 (3), 294-301. https://doi.org/10.1002/jcc.540040303.

(102) Martin, R. L. Natural Transition Orbitals. J. Chem. Phys. 2003, 118 (11), 4775-4777. https://doi.org/10.1063/1.1558471.

(103) Dunning, T. H. Gaussian Basis Sets for Use in Correlated Molecular Calculations. I. The Atoms Boron through Neon and Hydrogen. J. Chem. Phys. 1989, 90 (2), 1007-1023. https://doi.org/10.1063/1.456153.

(104) Kendall, R. A.; Dunning, T. H.; Harrison, R. J. Electron Affinities of the First-row Atoms Revisited. Systematic Basis Sets and Wave Functions. J. Chem. Phys. 1992, 96 (9), 67966806. https://doi.org/10.1063/1.462569.

(105) Gasser, M.; Bach, A.; Chen, P. Photodissociation Dynamics of the 2-Methylallyl Radical. Phys. Chem. Chem. Phys. 2008, No. 10, 1133-1138. https://doi.org/10.1063/1.479629.

(106) Gasser, M.; Schulenburg, A. M.; Dietiker, P. M.; Bach, A.; Merkt, F.; Chen, P. SinglePhoton and Resonance-Enhanced Multiphoton Threshold Ionization of the Allyl Radical. $J$. Chem. Phys. 2009, 131 (1), 0-8. https://doi.org/10.1063/1.3157185. 
(107) Wood, K. V; Taylor, J. W. A Photoionization Mass Spectrometric Study of Autoionization in Ethylene and Trans-2-Butene. Int. J. Mass Spectrom. Ion Phys. 1979, 30, 307-318.

(108) Schultz, T.; Fischer, I. The Nonradiative Decay of the Allyl Radical Excited $B^{2} A_{1}$ State Studied by Picosecond Time-Resolved Photoelectron Spectroscopy. J. Chem. Phys. 1997, 107 (19), 8197. https://doi.org/10.1063/1.475121.

(109) Schultz, T.; Clarke, J.; Gilbert, T.; Deyerl, H.; Fischer, I. Time- and Frequency-Resolved Photoionisation of the Allyl Radical. Faraday Discuss. 2000, No. 115, 17-31; discussion $79-102$.

(110) Schultz, T.; Fischer, I. Time-Resolved Photoelectron Spectroscopy of the Allyl Radical: The Lifetimes of the Ultraviolet Bands. J. Chem. Phys. 1998, 109 (14), 5812-5822. https://doi.org/10.1063/1.477203.

(111) Currie, C. L.; Ramsay, D. A. Electronic Absorption Spectrum and Dissociation Energy of the Allyl Radical. J. Chem. Phys. 1966, 45 (2), 488-491. https://doi.org/10.1063/1.1727594.

(112) Lee, A. M. D.; Coe, J. D.; Ullrich, S.; Ho, M. L.; Lee, S. J.; Cheng, B. M.; Zgierski, M. Z.; Chen, I.-C.; Martinez, T. J.; Stolow, A. Substituent Effects on Dynamics at Conical Intersections: $\alpha, \beta$-Enones. $J$. Phys. Chem. A 2007, 111 (47), 11948-11960. https://doi.org/10.1021/jp911286s.

(113) Neville, S. P.; Wang, Y.; Boguslavskiy, A. E.; Stolow, A.; Schuurman, M. S. Substituent Effects on Dynamics at Conical Intersections: Allene and Methyl Allenes. J. Chem. Phys. 2016, 144 (1), 1-16. https://doi.org/10.1063/1.4938561. 
(114) Wu, G.; Boguslavskiy, A. E.; Schalk, O.; Schuurman, M. S.; Stolow, A. Ultrafast NonAdiabatic Dynamics of Methyl Substituted Ethylenes: The П3s Rydberg State. J. Chem. Phys. 2011, 135 (16). https://doi.org/10.1063/1.3652966.

(115) Herterich, J.; Gerbich, T.; Fischer, I. Excited-State Dynamics of the 2-Methylallyl Radical. ChemPhysChem 2013, 14 (17), 3906-3908. https://doi.org/10.1002/cphc.201300700.

(116) Hemberger, P.; Köhler, J.; Fischer, I.; Piani, G.; Poisson, L.; Mestdagh, J.-M. Femtosecond Dynamics of Cyclopropenylidene, c- $\mathrm{C}_{3} \mathrm{H}_{2}$. Phys. Chem. Chem. Phys. 2012, 14 (18), $6173-$ 6178. https://doi.org/10.1039/C2CP23728H.

(117) Gosselin, J. L.; Weber, P. M. Rydberg Fingerprint Spectroscopy: A New Spectroscopic Tool with Local and Global Structural Sensitivity. J. Phys. Chem. A 2005, 109 (22), 4899 4904. https://doi.org/10.1021/jp0503866.

(118) Tsubouchi, M.; Suzuki, T. Femtosecond Photoelectron Imaging on Pyridine: Ultrafast Electronic Dephasing from the $\mathrm{S}_{1}\left(\mathrm{~N} \pi^{*}\right)$ State and Rydberg State Energetics. J. Phys. Chem. A 2003, 107 (50), 10897-10903. https://doi.org/10.1021/jp036333a.

(119) Schalk, O.; Boguslavskiy, A. E.; Schuurman, M. S.; Brogaard, R. Y.; Unterreiner, A. N.; Wrona-Piotrowicz, A.; Werstiuk, N. H.; Stolow, A. Substituent Effects on Dynamics at Conical Intersections: Cycloheptatrienes. J. Phys. Chem. A 2013, 117 (40), 10239-10247. https://doi.org/10.1021/jp309875m.

(120) Röder, A.; Humeniuk, A.; Giegerich, J.; Fischer, I.; Poisson, L.; Mitrić, R. Femtosecond Time-Resolved Photoelectron Spectroscopy of the Benzyl Radical. Phys. Chem. Chem. Phys. 2017, 19 (19), 12365-12374. https://doi.org/10.1039/C7CP01437F. 
(121) Steglich, M.; Knopp, G.; Hemberger, P. How the Methyl Group Position Influences the Ultrafast Deactivation in Aromatic Radicals. Phys. Chem. Chem. Phys. 2018. https://doi.org/10.1039/xxxxxxxx/.

(122) Murakami, Y.; Oguchi, T.; Hashimoto, K.; Nosaka, Y. Theoretical Study of the Benzyl + $\mathrm{O}_{2}$ Reaction: Kinetics, Mechanism and Product Branching Ratios. J. Phys. Chem. A. 2007, $111,13200-13208$.

(123) Sivaramakrishnan, R.; Michael, J. V. Pyrolysis of $\mathrm{C}_{6} \mathrm{D}_{5} \mathrm{CH}_{3}$ : Rate Constants and Branching Ratios in the High-Temperature Thermal Decomposition of Toluene. Proc. Combust. Inst. 2011, 33 (1), 225-232. https://doi.org/10.1016/j.proci.2010.05.077.

(124) Eng, R. A.; Gebert, A.; Goos, E.; Hippler, H.; Kachiani, C. Incubation Times, Fall-off and Branching Ratios in the Thermal Decomposition of Toluene: Experiments and Theory. Phys. Chem. Chem. Phys. 2002, 4 (16), 3989-3996. https://doi.org/10.1039/b202999e.

(125) Davis, W. M.; Heck, S. M.; Pritchard, H. O. Theoretical Study of Benzyl Radical Reactivity in Combustion Systems. J. Chem. Soc., Faraday Trans. 1998, 94 (September), 2725-2728.

(126) da Silva, G.; Cole, J. a; Bozzelli, J. W. Thermal Decomposition of the Benzyl Radical to Fulvenallene $\left(\mathrm{C}_{7} \mathrm{H}_{6}\right)+$ H. J. Phys. Chem. A 2009, 113 (21), 6111-6120. https://doi.org/10.1021/jp901933x.

(127) Derudi, M.; Polino, D.; Cavallotti, C. Toluene and Benzyl Decomposition Mechanisms: Elementary Reactions and Kinetic Simulations. Phys. Chem. Chem. Phys. 2011, 13 (48), 21308. https://doi.org/10.1039/c1cp22601k. 
(128) Carrington, A.; Smith, I. C. P. The Electron Spin Resonance Spectrum and Spin Density Distribution of the Benzyl Radical. Mol. Phys. 1965, 9 (2), 137-147. https://doi.org/10.1080/00268976500100151.

(129) Fateley, W. G.; Lippincott, E. R. Vibrational Spectrum and Structure of the Tropylium Ion. J. Chem. Phys. 1957, 26 (6), 1471-1481. https://doi.org/10.1063/1.1743566.

(130) Cotton, F. A. Chemical Applications of Group Theory. Wiley-Intersience: New York 1990.

(131) Jahn, H. A.; Teller, E. Stability of Polyatomic Molecules in Degenerate Electronic States. I. Orbital Degeneracy. Proc. R. Soc. Ser. A, Math. Phys. Sci. 1937, 161 (905), 220-235.

(132) Johnson, R. D. Excited Electronic States of the Tropyl (Cyclo- $\left.\mathrm{C}_{7} \mathrm{H}_{7}\right)$ Radical. J. Chem. Phys. 1991, 95 (10), 7108. https://doi.org/10.1063/1.461387.

(133) Satink, R. G.; Meijer, G.; Von Helden, G. Infrared Spectroscopy of Neutral $\mathrm{C}_{7} \mathrm{H}_{7}$ Isomers: Benzyl and Tropyl. J. Am. Chem. Soc. 2003, 125 (51), 15714-15715. https://doi.org/10.1021/ja038329i.

(134) Stakhursky, V. L.; Sioutis, I.; Tarczay, G.; Miller, T. A. Computational Investigation of the Jahn-Teller Effect in the Ground and Excited Electronic States of the Tropyl Radical. Part I. Theoretical Calculation of Spectroscopically Observable Parameters. J. Chem. Phys. 2008, 128 (8). https://doi.org/10.1063/1.2829437.

(135) Sioutis, I.; Stakhursky, V. L.; Tarczay, G.; Miller, T. A. Experimental Investigation of the Jahn-Teller Effect in the Ground and Excited Electronic States of the Tropyl Radical. Part II. Vibrational Analysis of the $\tilde{\mathrm{A}}^{2} \mathrm{E}_{3} "-\tilde{\mathrm{X}}^{2} \mathrm{E}_{2}$ " Electronic Transition. J. Chem. Phys. 2008, 
128 (8). https://doi.org/10.1063/1.2829471.

(136) Kaufmann, M.; Leicht, D.; Havenith, M.; Broderick, B. M.; Douberly, G. E. Infrared Spectroscopy of the Tropyl Radical in Helium Droplets. J. Phys. Chem. A 2016, 120 (34), 6768-6773. https://doi.org/10.1021/acs.jpca.6b06522.

(137) Silverstone, H. J.; Wood, D. E.; McConnell, H. M. Study of Molecular Orbital Degeneracy in $\mathrm{C}_{7} \mathrm{H}_{7}$. J. Chem. Phys. 1964, 41 (8), 2311-2323. https://doi.org/10.1063/1.1726265.

(138) Barney Ellison, G.; Davico, G. E.; Bierbaum, V. M.; DePuy, C. H. Thermochemistry of the Benzyl and Allyl Radicals and Ions. Int. J. Mass Spectrom. Ion Process. 1996, 156, 109131. https://doi.org/10.1016/S0168-1176(96)04383-2.

(139) Roth, W. R.; Hunold, F.; Neumann, M. Sauerstoff-Abfang von Radikalen in Uberkritischem Kohlendioxid; Bildungsenthalpie Und Rekombinationsbarriere Des CycloheptatrienylRadikals. 1996, 1679-1690.

(140) Lifshitz, C.; Gotkis, Y.; Ioffe, A.; Laskin, J.; Shaik, S. Is the Tropylium Ion (Tr+) Formed from Toluene at Its Thermochemical Threshold? Int. J. Mass Spectrom. Ion Process. 1993, 125, R7-R11. https://doi.org/10.1016/0168-1176(93)80020-F.

(141) Fischer, K. H.; Hemberger, P.; Bodi, A.; Fischer, I. Photoionisation of the Tropyl Radical. Beilstein J. Org. Chem. 2013, 9, 681-688. https://doi.org/10.3762/bjoc.9.77.

(142) Savee, J. D.; Zádor, J.; Hemberger, P.; Sztáray, B.; Bodi, A.; Osborn, D. L. Threshold Photoelectron Spectrum of the Benzyl Radical. Mol. Phys. 2015, 113 (15-16), 2217-2227. https://doi.org/10.1080/00268976.2015.1021398. 
(143) Fukushima, M. Dispersed Fluorescence Spectra from Single Vibronic Levels of the Benzyl Radical Observed in a Pulsed Discharge Jet. 1995, No. August.

(144) Uejoh, K. Fluorescence Spectrum of the Benzyl Radical in Methylcyclohexane at $4.2 \mathrm{~K}$. Spectrochim. Acta - Part A Mol. Biomol. Spectrosc. 2004, 60 (3), 595-602. https://doi.org/10.1016/S1386-1425(03)00267-1.

(145) Cossart-Magos, C.; Leach, S. Determination of the Symmetry of the First Excited Electronic State of Benzyl by Rotational Contour Analysis of Vibronic Bands of the Emission Spectra of $\mathrm{C}_{6} \mathrm{H}_{5} \mathrm{CH}_{2}, \mathrm{C}_{6} \mathrm{H}_{5} \mathrm{CD}_{2}$, and $\mathrm{C}_{6} . \quad J$. Chem. Phys. 1972, 56 (4), 1534-1545. https://doi.org/10.1063/1.1677403.

(146) Carrick, P. G.; Selco, J. I. Low Temperature Rotational Band Contours of Vibronic Transitions of the Benzyl Radical. J. Mol. Spectrosc. 1990, 139 (2), 449-452. https://doi.org/10.1016/0022-2852(90)90081-Z.

(147) Leach, S. Étude Des Spectres Électroniques de Molecules a Courte Durée de Vie, Par l'analyse Des Contours de Rotation de Bandes Vibroniques. Application Au Spectre d'émission Du Radical Benzyle. J. Phys. Colloq. 1971, 32 (C5), C5a-155-C5a-157.

(148) Miller, H.; Andrews, L. Emission and Excitation Spectra of Benzyl Radicals in Solid Argon. J. Mol. Spectrosc. 1981, 90, 20-26.

(149) Rice, J. E.; Handy, N. C.; Knowles, P. J. An MCSCF Study of the $\mathrm{X}^{2} \mathrm{~B}_{2},{ }^{2} \mathrm{~A}_{2}$, and $2{ }^{2} \mathrm{~B}_{2}$ States of Benzyl. J. Chem. Soc. Faraday Trans. 1987, 83 (9), 1643-1649.

(150) Maurice, D.; Head-gordon, M. On the Nature of Electronic Transitions in Radicals : An 
Extended Single Excitation Configuration Interaction Method. J. Phys. Chem. 1996, 100, $6131-6137$.

(151) Ward, B. Absorption Spectra of Aromatic Free Radicals: A Vibrational Analysis of the 3050 A Absorption Spectrum of Benzyl and a New Transition of Phenoxyl. Spectrochim. Acta. A. Mol. Biomol. Spectrosc. 1997, 24 (1966), 813-818.

(152) Margraf, M.; Noller, B.; Schröter, C.; Schultz, T.; Fischer, I. Time- and FrequencyResolved Photoionization of the $\mathrm{C}^{2} \mathrm{~A}_{2}$ State of the Benzyl Radical, $\mathrm{C}_{7} \mathrm{H}_{7}$. J. Chem. Phys. 2010, 133 (7). https://doi.org/10.1063/1.3469787.

(153) Ikeda, N.; Nakashima, N.; Yoshihara, K. Formation and Relaxation of Hot Benzyl Radicals in the Gas Phase. J. Phys. Chem. 1984, 88 (24), 5803-5806. https://doi.org/10.1021/j150668a013.

(154) Pino, T.; Güthe, F.; Ding, H.; Maier, J. P. Gas-Phase Electronic Spectrum of the Tropyl $\mathrm{C}_{7} \mathrm{H}_{7} \quad$ Radical. J. Phys. Chem. A 2002, $106 \quad$ (42), $10022-10026$. https://doi.org/10.1021/jp020910y.

(155) Thrush, B. A.; Zwolenik, J. J. Spectrum of the Tropyl Radical. Discuss. Faraday Soc. 1963, 35, 196. https://doi.org/10.1039/df9633500196.

(156) Knowles, P. J.; Werner, H. J. An Efficient Method for the Evaluation of Coupling Coefficients in Configuration Interaction Calculations. Chem. Phys. Lett. 1988, 145 (6), 514-522. https://doi.org/10.1016/0009-2614(88)87412-8.

(157) Werner, H.-J.; Knowles, P. J.; Knizia, G.; Manby, F. R.; Schütz, M. MOLPRO,Version 
2012.1, a Package of Ab Initio Programs. 2012.

(158) Werner, H. J.; Knowles, P. J. An Efficient Internally Contracted MulticonfigurationReference Configuration Interaction Method. J. Chem. Phys. 1988, 89 (9), 5803-5814. https://doi.org/10.1063/1.455556.

(159) Zierhut, M.; Noller, B.; Schultz, T.; Fischer, I. Excited-State Decay of Hydrocarbon Radicals, Investigated by Femtosecond Time-Resolved Photoionization: Ethyl, Propargyl, and Benzyl. J. Chem. Phys. 2005, 122 (9), 1-8. https://doi.org/10.1063/1.1857475.

(160) Song, Y.; Zheng, X.; Lucas, M.; Zhang, J. Ultraviolet Photodissociation Dynamics of the Benzyl Radical. Phys. Chem. Chem. Phys. 2011, 13 (18), 8296. https://doi.org/10.1039/c1cp20310j.

(161) Tully, J. C. Molecular Dynamics with Electronic Transitions. J. Chem. Phys. 1990, 93 (2), 1061-1071. https://doi.org/10.1063/1.459170.

(162) Doering, W. V. E.; Knox, L. H.; Doering, W. V. E. Reactions of the Cycloheptatrienylium (Tropylium) Ion. J. Am. Chem. Soc. $\quad$ 1957, $79 \quad$ (2), 352-356. https://doi.org/10.1021/ja01559a030.

(163) Elder, F. A. Photoionization of the Cycloheptatrienyl Radical. J. Chem. Phys. 1969, 50 (2), 1027. https://doi.org/10.1063/1.1671081.

(164) Harrison, A. G.; Honnen, L. R.; Dauben, H. J.; Lossing, F. P. Free Radicals by Mass Spectrometry. XX. Ionization Potentials of Cyclopentadienyl and Cycloheptatrienyl Radicals. $\quad J . \quad$ Am. $\quad$ Chem. Soc. $\quad$ 1960, $82 \quad$ (21), 5593-5598. 
https://doi.org/10.1021/ja01506a008.

(165) Gloaguen, E.; Mestdagh, J. M.; Poisson, L.; Lepetit, F.; Visticot, J. P.; Soep, B.; Coroiu, M.; Eppink, A. T. J. B.; Parker, D. H. Experimental Evidence for Ultrafast Electronic Relaxation in Molecules, Mediated by Diffuse States. J. Am. Chem. Soc. 2005, 127 (47), 16529-16534. https://doi.org/10.1021/ja052269u.

(166) Flynn, C. R.; Michl, J. $\pi$, $\pi$-Biradicaloid Hydrocarbons: o-Xylylene. Photochemical Preparation from 1,4-Dihydrophthalazine in Rigid Glass, Electric Spectroscopy, and Calculations. J. Am. Chem. Soc. 1974, 96 (10), 3280-3288. https://doi.org/10.1021/ja00817a042.

(167) Mahaffy, P. G.; Wieser, J. D.; Montgomery, L. K. An Electron Diffraction Study of PXylylene. J. Am. Chem. Soc. 1977, 99 (13), 4514-4515. https://doi.org/10.1021/ja00455a057.

(168) Pearson, J. M.; Six, H. A.; Williams, D. J.; Levy, M. Spectroscopic Studies of Quinodimethanes. J. Am. Chem. Soc. 1971, 93 (20), 5034-5036. https://doi.org/10.1021/ja00749a010.

(169) Coulson, C. A.; Craig, D. P.; Maccoll, A.; Pullman, M. A. P-Quinodimethane and Its Diradical. J. Am. Chem. Soc. 1942, 64, 36-38.

(170) Bobrowski, M.; Skurski, P.; Freza, S. The Electronic Structure of P-Xylylene and Its Reactivity with Vinyl Molecules. Chem. Phys. 2011, 382 (1-3), 20-26. https://doi.org/10.1016/j.chemphys.2011.02.003. 
(171) Ha, T. An Ab Initio Study of the Ground and Excited States of p-Benzoquinone. Theor. Chim. Acta 1975, 36 (4), 345. https://doi.org/10.1016/0301-0104(85)87101-9.

(172) Koenig, T.; Wielesek, R.; Snell, W.; Baile, T. Helium(I) Photoelectron Spectrum of pQuinodimethane. J. Am. Chem. Soc. 1975, $97 \quad$ (11), 3225-3226. https://doi.org/10.1021/ja00844a055.

(173) Hemberger, P.; Trevitt, A. J.; Ross, E.; Da Silva, G. Direct Observation of Para-Xylylene as the Decomposition Product of the Meta-Xylyl Radical Using VUV Synchrotron Radiation. J. Phys. Chem. Lett. 2013, 4 (15), 2546-2550. https://doi.org/10.1021/jz401207z.

(174) Watanabe, K. Photoionization and Total Absorption Cross Section of Gases. I. Ionization Potentials of Several Molecules. Cross Sections of NH3 and NO. J. Chem. Phys. 1954, 22 (9), 1564-1570. https://doi.org/10.1063/1.1740459.

(175) Issler, K.; Röder, A.; Hirsch, F.; Poisson, L.; Fischer, I.; Mitrić, R.; Petersen, J. Excited State Dynamics and Time-Resolved Photoelectron Spectroscopy of Para-Xylylene. Faraday Discuss. 2018, 212, 83-100.

(176) Koslowski, A.; Beck, M. E.; Thiel, W. Implementation of a General Multireference Configuration Interaction Procedure with Analytic Gradients in a Semiempirical Context Using the Graphical Unitary Group Approach. J. Comput. Chem. 2003, 24 (6), 714-726. https://doi.org/10.1002/jcc.10210.

(177) Patchkovskii, S.; Koslowski, A.; Thiel, W. Generic Implementation of Semi-Analytical CI Gradients for NDDO-Type Methods. Theor. Chem. Acc. 2005, 114 (1-3), 84-89. 
https://doi.org/10.1007/s00214-005-0647-y.

(178) Scholten, M. Semiempirical Methods with Orthogonalization Corrections: The OM3 Method, Universität Düsseldorf, Germany, 2003.

(179) Weber, W.; Thiel, W. Orthogonalization Corrections for Semiempirical Methods. Theor. Chem. Acc. 2000, 103 (6), 495-506. https://doi.org/10.1007/s002149900083.

(180) Döhnert, D.; Koutecky, J. Occupation Numbers of Natural Orbitals as a Criterion for Biradical Character. Different Kinds of Biradicals. J. Am. Chem. Soc. 1980, 102 (6), 17891796. https://doi.org/10.1021/ja00526a005.

(181) Thaddeus, P.; Gottlieb, C. A.; Mollaaghababa, R.; Vrtilek, J. M. Free Carbenes in the Interstellar Gas. J. Chem. Soc. Faraday Trans. 1993, 89 (13), 2125. https://doi.org/10.1039/ft9938902125.

(182) Vrtilek, J. M.; Gottlieb, C. A.; Thaddeus, P. Laboratory and Astronomicals Pectroscopy of $\mathrm{C}_{3} \mathrm{H}_{2}$, the First Interstellar Organic Ring. Astrophys. J. 1987, 314, 716-725.

(183) Holzmeier, F.; Fischer, I.; Kiendl, B.; Krueger, A.; Bodi, A.; Hemberger, P. On the Absolute Photoionization Cross Section and Dissociative Photoionization of Cyclopropenylidene. Phys. Chem. Chem. Phys. 2016, 18 (13), 9240-9247. https://doi.org/10.1039/c6cp01068g.

(184) Hemberger, P.; Noller, B.; Steinbauer, M.; Fischer, K.; Fischer, I. The B ${ }^{1} B_{1}$ State of Cyclopropenylidene, $\quad \mathrm{c}_{3} \mathrm{C}_{3} \mathrm{H}_{2} . \quad J . \quad$ Phys. Chem. Lett. 2010, 1 (1), 228-231. https://doi.org/10.1021/jz900114r.

(185) Schuurman, M. S.; Giegerich, J.; Pachner, K.; Lang, D.; Kiendl, B.; MacDonell, R. J.; 
Krueger, A.; Fischer, I. Photodissociation Dynamics of Cyclopropenylidene, c- $\mathrm{C}_{3} \mathrm{H}_{2}$. Chem. - A Eur. J. 2015, 21 (41), 14486-14495. https://doi.org/10.1002/chem.201501624.

(186) Giegerich, J.; Fischer, I. The Photodissociation Dynamics of Alkyl Radicals. J. Chem. Phys. 2015, 142 (4). https://doi.org/10.1063/1.4906605.

(187) Negru, B.; Just, G. M. P.; Park, D.; Neumark, D. M. Photodissociation Dynamics of the Tert-Butyl Radical via Photofragment Translational Spectroscopy at 248 Nm. Phys. Chem. Chem. Phys. 2011, 13 (18), 8180. https://doi.org/10.1039/c0cp02461a.

(188) Lengsfield, B. H.; Siegbahn, P. E. M.; Liu, B. Ab Initio Assignment of the UV Spectra of the Ethyl, Isopropyl, and t-Butyl Radicals. J. Chem. Phys. 1984, 81 (2), 710-716. https://doi.org/10.1063/1.447754.

(189) Noller, B.; Maksimenka, R.; Fischer, I.; Armone, M.; Engels, B.; Alcaraz, C.; Poisson, L.; Mestdagh, J. Femtosecond Dynamics of the Tert -Butyl Radical , t-C44. $\mathrm{H}_{9}$. J. Phys. Chem. A 2007, 111, 1771-1779.

(190) Song, J. K.; Tsubouchi, M.; Suzuki, T. Femtosecond Photoelectron Imaging on Pyrazine: Spectroscopy of 3s and 3p Rydberg States. J. Chem. Phys. 2001, 115 (19), 8810. https://doi.org/10.1063/1.1410974.

(191) Zierhut, M.; Roth, W.; Fischer, I. Photodissociation of the Tert-Butyl Radical, $\mathrm{C}_{4} \mathrm{H}_{9} . J$. Phys. Chem. A 2004, 108 (39), 8125-8130. https://doi.org/10.1021/jp048792x.

(192) Wendt, H. R.; Hunziker, H. E. The UV Spectra of Primary, Secondary, and Tertiary Alkyl Radicals. J. Chem. Phys. 1984, 81 (2), 717-723. https://doi.org/10.1063/1.447755. 
(193) Lee, S.-H.; Tang, K.-C.; Chen, I.-C.; Schmitt, M.; Shaffer, J. P.; Schultz, T.; Underwood, J. G.; Zgierski, M. Z.; Stolow, A. Substituent Effects in Molecular Electronic Relaxation Dynamics via Time-Resolved Photoelectron Spectroscopy: $\pi \pi^{*}$ States in Benzenes. J. Phys. Chem. A 2002, 106 (39), 8979-8991. https://doi.org/10.1021/jp021096h.

(194) Kholghy, M. R.; Kelesidis, G. A.; Pratsinis, S. E. Reactive Polycyclic Aromatic Hydrocarbon Dimerization Drives Soot Nucleation. Phys. Chem. Chem. Phys. 2018, 20 (16), 10925-10938. https://doi.org/10.1039/c7cp07803j.

(195) Mathur, R. B.; Singh, B. P.; Pande, S. Carbon Nanomaterials Synthesis, Structure, Properties and Applications, 1st Editio.; Taylor \& Francis, 2016.

(196) Sakai, N.; Yamamoto, S. Warm Carbon-Chain Chemistry. Chem. Rev. 2013, 113 (12), 8981-9015. https://doi.org/10.1021/cr4001308.

(197) Adachi, S.; Kohguchi, H.; Suzuki, T. Unravelling the Electronic State of $\mathrm{NO}_{2}$ Product in Ultrafast Photodissociation of Nitromethane. J. Phys. Chem. Lett. 2018, 9 (2), 270-273. https://doi.org/10.1021/acs.jpclett.7b03032.

(198) Forbes, R.; Makhija, V.; Veyrinas, K.; Stolow, A.; Lee, J. W. L.; Burt, M.; Brouard, M.; Vallance, C.; Wilkinson, I.; Lausten, R.; et al. Time-Resolved Multi-Mass Ion Imaging: Femtosecond UV-VUV Pump-Probe Spectroscopy with the PImMS Camera. J. Chem. Phys. 2017, 147 (1), 013911. https://doi.org/10.1063/1.4978923.

(199) Vincow, G.; Dauben, H. J.; Hunter, F. R.; Volland, W. V. Resonance Energy of Cycloheptatrienyl Radical. J. Am. Chem. Soc. 1969, 91 (11), 2823-2827. https://doi.org/10.1021/ja01039a001. 
TOC graphic

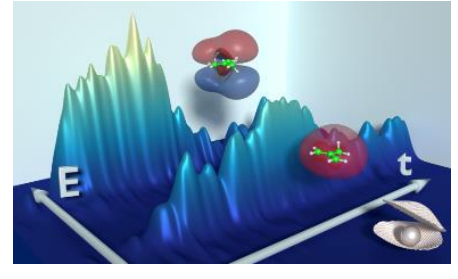

